\title{
Nonlinear optical response of doped mono- and bilayer graphene: length gauge tight-binding model
}

\author{
F. Hipolito, ${ }^{1, *}$ Alireza Taghizadeh, ${ }^{1}$ and T. G. Pedersen ${ }^{1,2, \dagger}$ \\ ${ }^{1}$ Department of Physics and Nanotechnology, Aalborg University, DK-9220 Aalborg Øst, Denmark \\ ${ }^{2}$ Center for Nanostructured Graphene (CNG), DK-9220 Aalborg Øst, Denmark
}

\begin{abstract}
We compute the nonlinear optical response of doped mono- and bilayer graphene using the full dispersion based on tight-binding models. The response is derived with the density matrix formalism using the length gauge and is valid for any periodic system, with arbitrary doping. By collecting terms that define effective nonlinear response tensors, we identify all nonlinear Drude-like terms (up to third-order) and show that all additional spurious divergences present in the induced current vanish. The nonlinear response of graphene comprises a large Drude-like divergence and three resonances that are tightly connected with transitions occurring in the vicinity of the Fermi level. The analytic solution derived using the Dirac approximation captures accurately the firstand third-order responses in graphene, even at very high doping levels. The quadratic response of gapped graphene is also strongly enhanced by doping, even for systems with small gaps such as commensurate structures of graphene on $\mathrm{SiC}$. The nonlinear response of bilayer graphene is significantly richer, combining the resonances that stem from doping with its intrinsic strong low-energy resonances.
\end{abstract}

PACS numbers: 42.65.An,78.67.-n,78.67.Wj,81.05.ue

\section{INTRODUCTION}

The interaction of intense light with matter encompasses a wide range of phenomena with many applications in nonlinear optics, ${ }^{1,2}$ over a large portion of the energy spectrum. Recent developments in the production and characterization of 2D materials have led to intense experimental study of nonlinear optical phenomena, including multiple wave mixing processes, ${ }^{3-9}$ harmonic generation, ${ }^{10-22}$ and optical rectification $(\mathrm{OR}){ }^{23}$ From a theoretical point of view, several methods can be used to evaluate the nonlinear optical response functions, but frequently generate contradictory results, as discussed in Ref. 24 and 25. Several of these differences can be traced to approximations in the calculations, such as the truncation of the Hamiltonian basis size, or the choice of gauge. ${ }^{24-26}$ Methods derived using the length gauge (LG) have been shown to be least sensitive to the truncation of the basis set compared to the conventional velocity gauge (VG). ${ }^{24}$ Hence, the LG offers an accurate estimate of the nonlinear response even in calculations truncated to just two bands. In addition, the choice of response function can also lead to different results, as identified in Refs. 24 and 26. For instance, Ref. 26 shows that a direct evaluation of the third-order current density response function of cold insulators is plagued by several unphysical divergences, while the polarization density counterpart is regular.

In the present work, we compute the linear and nonlinear optical response of doped mono- and bilayer graphene using the LG formalism. For the third-order nonlinearity in monolayer graphene (MLG), we compare results obtained from the full band structure to those found within the Dirac approximation. We begin by deriving expressions for the current density response showing that the unphysical divergences in the response of cold insulators ${ }^{26}$ are spurious and can be removed for all nonlinear processes up to third-order, independently of the symmetry of the crystal. Moreover, we expand our previous formalism in Ref. 27 at several levels. First, we consider the single-particle nonlinear response valid for general crystalline systems with an arbitrary number of bands, rather than particular solutions valid for two-band models. Second, the expressions shown in the present work are valid for any wave mixing process up to third-order, rather than just the particular case of third harmonic generation (THG). Third, we demonstrate how, for arbitrary doped or intrinsic systems, it is possible to remove all spurious divergences previously observed in the evaluation of the conductivity tensor for cold insulators ${ }^{26}$ and identify nonlinear features analogous to the Drude peak that should be present in the response of doped semiconductors or metallic systems. Within the Dirac approximation, the first- and thirdorder LG results for MLG can be evaluated analytically and reproduce the previously identified logarithmic divergences. ${ }^{28-30}$ By comparison with full dispersion tight-binding (TB), the Dirac approximation is shown to accurately capture the third-order response, even for highly doped systems, with Fermi level up to $\mu=1.5 \mathrm{eV}$, that exceed current experimental reports.

We provide general expressions for nonlinear optical response using the LG and gauge invariant generalized derivatives, that are not limited by: i) particular solutions tailored for two-band systems, such as the Dirac approximation for MLG, truncated Hamiltonians for biased bilayer graphene (BBG); ii) cold semiconductors approximations; iii) lattice symmetry restrictions. Considering full dispersion TB models, our expressions can be used to probe the optical response at higher energies, including transitions with bands farther away from the Fermi level, of paramount importance to the nonlinear 
response of BBG, even at the energy scale of $\hbar \omega \sim 150$ $\mathrm{meV}$. All even-order nonlinear response functions vanish (in the dipole approximation) in centrosymmetric structures. A dipole-allowed even-order response in graphenebased systems can be obtained by e.g. rolling the material into a chiral nanotube. ${ }^{31}$ Instead, in the present study, we consider commensurate structures of graphene on $\mathrm{SiC}$ or hBN substrates. ${ }^{32-34}$ Such substrates break the sublattice symmetry of the two atoms in the graphene unitcell leading to broken centrosymmetry and opening of a band gap at the Dirac points. However, regardless of the broken symmetry the Dirac approximation still predicts vanishing even-order responses due to the full rotation symmetry of the Dirac Hamiltonian. ${ }^{35}$ In contrast, by using TB models that capture the reduced symmetry, our expressions can be used to correctly evaluate the quadratic response of such gapped systems.

We present results for the optical conductivity, THG, optical Kerr effect, and second harmonic generation (SHG). Regarding the latter, we consider the effect of doping on the quadratic response of non-centrosymmetric systems, such as graphene on $\mathrm{SiC}$ or $\mathrm{hBN}$ substrates $^{32-34}$ and hydrogenated graphene. ${ }^{36-38}$

\section{THEORETICAL FRAMEWORK}

The interaction of light with the electrons is treated in the dipole approximation and we, therefore, ignore the position-dependence of the electromagnetic field. We do not consider electron-electron interaction, i.e. excitonic effects, and therefore the many-body effects arise from the Fermi-Dirac statistics only. Hence, the total singleparticle Hamiltonian reads

$$
\hat{\mathcal{H}}=\hat{\mathcal{H}}_{0}+e \mathbf{r} \cdot \mathbf{E}(t)
$$

where $\hat{\mathcal{H}}_{0}$ denotes the unperturbed Hamiltonian of the crystal and $e>0$ is the elementary charge. The electromagnetic field $\mathbf{E}(t)$ is a linear combination of monochromatic fields restricted to propagate along the $z$ axis (normal to the crystal plane)

$$
\mathbf{E}(t)=\sum_{\alpha, \omega_{i}}\left[E_{\omega_{i}}^{\alpha} e^{-i \bar{\omega}_{i} t}+E_{-\omega_{i}}^{\alpha} e^{i \bar{\omega}_{i}^{*} t}\right] \mathbf{e}_{\alpha} / 2,
$$

where the polarization plane is taken as the $x O y$ plane. Throughout this paper, sub- or superscripts using the greek alphabet $\{\alpha, \beta, \lambda, \phi\}$ represent the spatial coordinates $\{x, y, z\}$. Furthermore, the adiabatic coupling of the interaction is ensured by the analytic continuation of the photon frequency $\bar{\omega} \equiv \omega+i \eta .{ }^{39}$ The diagonalization of the unperturbed periodic Hamiltonian provides the crystal band dispersions $\epsilon_{m}(\mathbf{k})$ and respective eigenstates $|m \mathbf{k}\rangle$, which serve as the basis for the calculation of the response function. Here, $m$ and $\mathbf{k}$ denote band index and electron wave vector, respectively. The calculation is based on the time-dependent density matrix, $\hat{\rho}(t) \equiv \sum_{\mathbf{k}} \sum_{m n} \rho_{m n}(\mathbf{k})|m \mathbf{k}\rangle\langle n \mathbf{k}|$, that obeys the quantum Liouville equation $i \hbar \partial \hat{\rho} / \partial t=[\hat{H}, \hat{\rho}]$, which lends itself to a perturbative expansion.

Excitonic effects have been shown to play an important role in the optical response of $2 \mathrm{D}$ materials, particularly in systems with large gaps such as hexagonal boron-nitride $(\mathrm{hBN}),{ }^{40-42}$ the vast class of transition-metal dichalcogenides, ${ }^{41,43-48}$ few-layered black phosphorus, ${ }^{47,49,50}$ and many others. Excitonic effects in the optical response usually manifest themselves as a red-shift of the response onset and significant transfer of spectral weight to bound excitons. In pristine suspended graphene, the electron-electron interaction can be important due to low screening of interactions, leading to potentially significant excitonic effects on the spectrum of graphene and on its low-energy linear ${ }^{51-61}$ and nonlinear ${ }^{62,63}$ optical response. Conversely, the presence of substrates or encapsulation of graphene increases the screening of interactions rendering the excitonic effects negligible for MLG and in commensurate systems of graphene on $\mathrm{SiC}$ or hBN. Moreover, electron-electron interactions can cause additional effects, such as the renormalization of the low-energy band structure, ${ }^{51,64,65}$ leading to further corrections to the low energy optical response. ${ }^{54-57,59,65-67}$

In $\mathrm{BBG}$, the potentially larger gaps can give rise to moderate manifestations of excitonic coupling 68,69 in charge neutral systems. The presence of free carriers in doped systems prompts strong screening of electronelectron interactions, and consequently the manifestations of excitonic effects should be at least softened, if not removed altogether. Therefore, single-particle calculations of the response of doped MLG and BBG offer a sound description of the optical response. ${ }^{70-75}$

\section{A. Optical response of multi-band systems}

We evaluate the optical response to an external electromagnetic field based on the current density response $\mathbf{J}(t)=\operatorname{Tr}[\hat{\rho}(t) \hat{\mathbf{j}}]$. The current density operator $\hat{\mathbf{j}} \equiv$ $-g e \hat{\mathbf{v}} / \Omega$ is then defined in terms of the single-particle velocity $\hat{\mathbf{v}}=i\left[\hat{H}_{0}, \hat{\mathbf{r}}\right] / \hbar$, spin degeneracy $g=2$, and the $D$-dimensional volume of the system $\Omega$. The integration of the equation of motion of the density matrix is based on the LG formalism proposed in Ref. 26. In this approach, the equation of motion of the density matrix reads

$$
\begin{aligned}
i \hbar \frac{\partial \rho_{m n}}{\partial t} & =\epsilon_{m n} \rho_{m n}+i e\left(\rho_{m n}\right)_{; \mathbf{k}} \cdot \mathbf{E}(t) \\
& +e \sum_{l}\left[\bar{\delta}_{m l} \mathcal{A}_{m l} \rho_{l n}-\bar{\delta}_{l n} \mathcal{A}_{l n} \rho_{m l}\right] \cdot \mathbf{E}(t),
\end{aligned}
$$

where we make use of the "generalized derivative" $\left(S_{m n}\right)_{; k_{\alpha}}=\left(S_{m n}\right)_{; \alpha} \equiv \partial S_{m n} / \partial k_{\alpha}-i S_{m n}\left(\mathcal{A}_{m m}^{\alpha}-\mathcal{A}_{n n}^{\alpha}\right)$, $\bar{\delta}_{m n} \equiv 1-\delta_{m n}$, and the energy dispersion differences $\epsilon_{m n} \equiv \epsilon_{m}-\epsilon_{n}$. To simply notation, we omit the explicit 
$\mathbf{k}$ dependence on all variables, e.g. the density matrix $\rho_{m n} \equiv \rho_{m n}(\mathbf{k})$. Moreover, the matrix elements of the Berry connection in periodic systems read

$$
\mathcal{A}_{m n}=\frac{i}{\Omega_{C}} \int_{\Omega_{C}} \mathrm{~d} \mathbf{r} u_{m \mathbf{k}}^{*}(\mathbf{r}) \nabla_{\mathbf{k}} u_{n \mathbf{k}}(\mathbf{r})
$$

with cell-periodic functions $u_{m \mathbf{k}}(\mathbf{r})^{26,76}$ and cell volume $\Omega_{C}$. For details regarding calculation of the perturbative solution for the density matrix we refer to the extensive literature. ${ }^{24,26-29,42,76-82}$ We follow the procedure and notation outlined in Ref. 76 and present the relevant results for the first-, second-, and third-order terms of the density matrix in Eqs. (A1a), (A1b), and (A1c), respectively. We then find the optical response (linear and nonlinear) by evaluating the $n^{\text {th }}$-order current density

$$
\begin{aligned}
j_{\phi}^{(n)}(t) & =\sum_{\omega_{n} \ldots \omega_{1}} \sum_{\lambda \ldots \alpha} \sigma_{\phi \lambda \ldots \alpha}^{(n)}\left(\omega_{n}+\ldots+\omega_{1}\right) \\
& \times E_{\omega_{n}}^{\lambda} \ldots E_{\omega_{1}}^{\alpha} e^{-i\left(\bar{\omega}_{n}+\ldots+\bar{\omega}_{1}\right) t} .
\end{aligned}
$$

The final expressions for the response functions are rather cumbersome containing various combinations of intraband $(i)$ and interband $(e)$ transitions. We consequently relegate the full expressions to the appendix, in which conductivities up to third-order can be found in Eqs. (A2), (A3), and (A4).

\section{B. $\pi$-electron tight-binding}

The low-energy electronic properties of graphene systems with an underlying honeycomb lattice, see Fig. 1a, can be characterized by orthogonal TB models that include a $p_{z}$ orbital per atom in the unit-cell. In this context, the Hamiltonian operator $\hat{H}=\sum_{\mathbf{k}} \Psi_{\mathbf{k}}^{\dagger} h_{\mathbf{k}} \Psi_{\mathbf{k}}$ lends itself to a simple representation in crystal momentum, where $\Psi_{\mathrm{k}}^{\dagger}$ represents the Fourier-transformed electron creation operators at different sites in the unit cell. The Fourier transforms of the Hamiltonians for MLG and AB-stacked BBG read

$$
\begin{aligned}
h_{\mathbf{k}}^{\mathrm{MLG}} & \equiv\left(\begin{array}{cc}
-\delta / 2 & \gamma_{0} f \\
\gamma_{0} f^{*} & \delta / 2
\end{array}\right), \\
h_{\mathbf{k}}^{\mathrm{BBG}} & \equiv\left(\begin{array}{cccc}
-\Delta / 2 & \gamma_{0} f & \gamma_{4} f & \gamma_{3} f^{*} \\
\gamma_{0} f^{*} & -\Delta / 2 & \gamma_{1} & \gamma_{4} f \\
\gamma_{4} f^{*} & \gamma_{1} & \Delta / 2 & \gamma_{0} f \\
\gamma_{3} f & \gamma_{4} f^{*} & \gamma_{0} f^{*} & \Delta / 2
\end{array}\right),
\end{aligned}
$$

where $f \equiv \exp \left(i k_{y} a_{0}\right)+2 \exp \left(-i k_{y} a_{0} / 2\right) \cos \left(\sqrt{3} k_{x} a_{0} / 2\right)$, and nearest neighbor distance $a_{0}=1.42 \AA$. The hopping integral for graphene is taken as $\gamma_{0}=-3.0$ $\mathrm{eV}^{75,83-85}$ while parametrization of TB models for the $\mathrm{AB}$ stacked bilayer graphene has been an issue of intense research. ${ }^{75,77,83-92}$ In this work, we follow the parametrization obtained from ARPES data in Ref. 77: $\gamma_{0}=-3.21 \mathrm{eV}, \gamma_{1}=0.61 \mathrm{eV}, \gamma_{3}=0.39 \mathrm{eV}$, and $\gamma_{4}=0.15 \mathrm{eV}$, where $\gamma_{1}, \gamma_{3}$ and $\gamma_{4}$ are the interlayer hopping integrals. The presence of an on-site potential $\delta$ in (a)
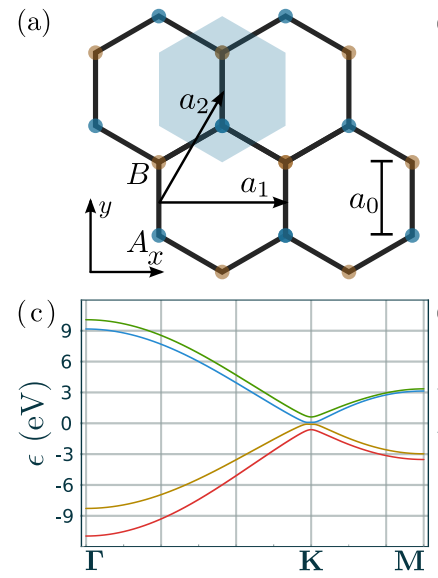

(b)
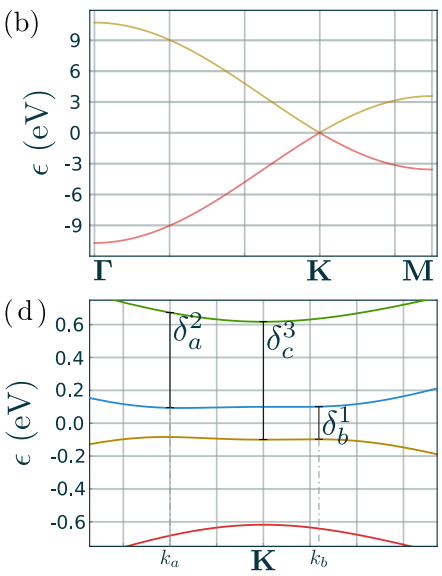

FIG. 1. (a) Representation of the monolayer lattice, including the primitive vectors and Wigner-Seitz cell (light blue hexagon). Energy dispersions for MLG (b), and BBG (c) and (d). Plots (c) and (d) consider BBG with external bias potential $\Delta=200 \mathrm{meV}$. In (d), we indicate some of the energy differences $\delta_{l}^{i}$ occurring at the van Hove singularities (vHs) between the $i$ th pair of bands, as listed in Tab. I.

TABLE I. Energy differences $\delta_{l}^{i}$ between the $i$ th pair of bands $(m, n)$ at the $k_{l}$ vHs in the BBG, with external bias potential set at $\Delta=200 \mathrm{meV}$. The three relevant low-energy vHs are found along the high symmetry paths $\overline{\mathbf{\Gamma K}}$ and $\overline{\mathbf{K M}}$, respectively $k_{a}$ and $k_{b}$, and at the high symmetry point $\mathbf{K}=k_{c}$, as shown in Fig. 1d.

\begin{tabular}{cccc}
\hline \hline$i$ & 1 & 2 & 3 \\
$(m, n)$ & $(3,2)$ & $(4,3)$ & $(4,2)$ \\
\hline$\delta_{a}^{i}(\mathrm{eV})$ & 0.178 & 0.585 & 0.763 \\
$\delta_{b}^{i}(\mathrm{eV})$ & 0.198 & 0.534 & 0.731 \\
$\delta_{c}^{i}(\mathrm{eV})$ & 0.200 & 0.518 & 0.718 \\
\hline \hline
\end{tabular}

MLG and an interlayer bias $\Delta$ in BBG opens a gap in the respective energy dispersions. Estimates range from very small gaps for graphene-hBN structures and substrates, $E_{g}=31 \mathrm{meV}$, to larger $\sim 260 \mathrm{meV}$ in $\mathrm{SiC}$ substrates and $\sim 500$ in hydrogenated graphene. ${ }^{32,37}$ Hence, for pristine MLG we take $\delta=0$, while $\delta=E_{g}$ in the gapped case. In BBG, the gap can be tuned by electrostatic gating, ${ }^{11,93}$ providing an additional mechanism to control the optical properties of the material. In Figs. $1 b$ and 1c, we show the band structures along the relevant high symmetry paths. For the bilayer, we display the energy dispersion for a biased system $(\Delta=200 \mathrm{meV})$, highlighting the possible vertical transitions occurring at the low-energy van Hove singularities (vHs). The trigonal warping of the dispersion is amplified by the presence of finite interlayer hopping integrals $\gamma_{3}$ and $\gamma_{4}$ that shift the band gap along the high symmetry path $\overline{\boldsymbol{\Gamma K}}$. As a reference, the bias $\Delta$ dependent energy differences highlighted in Fig. 1d are listed in Tab. I.

The adoption of the crystal momentum representation simplifies the evaluation of matrix elements of the veloc- 
ity operator that reduce to $\mathbf{v}_{m n}=\hbar^{-1}\left\langle m \mathbf{k}\left|\nabla_{\mathbf{k}} h_{\mathbf{k}}\right| n \mathbf{k}\right\rangle$. Finally, the Berry connection in periodic systems ${ }^{76,94}$ reads $\mathcal{A}_{m n}=i\left\langle m \mathbf{k}\left|\boldsymbol{\nabla}_{\mathbf{k}}\right| n \mathbf{k}\right\rangle$. The details regarding the numerical implementation of the derivatives present in the Berry connection are discussed in Ref. 27 and references therein.

\section{Effective rank-2 tensors for the nonlinear response}

Here, we show that the spurious divergences found in the current density response ${ }^{26}$ naturally vanish when considering an effective rank-2 tensor for the nonlinear conductivity, rather than the conductivity tensor as defined in Eq. (5) or its susceptibility counterpart. The definition of effective tensors is not new per se, in fact, it has been used extensively in nonlinear optics, ${ }^{2,95,96}$ but is frequently not taken into consideration in theoretical calculations of the nonlinear response, where authors tend to only consider individual tensor elements. By combining and adding contributions according to the dependence on the fields and the output frequency, it is possible to define an effective rank-2 tensor $\bar{\sigma}_{\phi \nu}^{(n)}\left(\omega_{s}\right)$ for the $n^{\text {th }}$-order conductivity

$$
\begin{aligned}
j_{\phi}^{(n)}(t) & =\sum_{\omega_{n} \ldots \omega_{1}} \sum_{\lambda \ldots \alpha} \sigma_{\phi \lambda \ldots \alpha}^{(n)}\left(\omega_{n}, \ldots, \omega_{1}\right) E_{\omega_{n}}^{\lambda} \ldots E_{\omega_{1}}^{\alpha} \\
& \times \exp \left[-i\left(\bar{\omega}_{n}+\ldots+\bar{\omega}_{1}\right) t\right] \\
& \equiv \sum_{\omega_{s}} \sum_{\nu} \bar{\sigma}_{\phi \nu}^{(n)}\left(\omega_{s}\right) \mathcal{E}_{\omega_{s}}^{(\nu)} e^{-i \bar{\omega}_{s} t}
\end{aligned}
$$

where the index $\nu$ contains all combinations of $\lambda \ldots \alpha$ that preserve equal powers of the Cartesian components of the electric field, $\mathcal{E}_{\omega_{s}}^{(\nu)} \equiv E_{\omega_{n}}^{\lambda} \ldots E_{\omega_{1}}^{\alpha}$, with $\omega_{s} \equiv$ $\omega_{n}+\ldots+\omega_{1}$ defining the sum of all input frequencies. Several labeling conventions are possible for the effective tensor elements. ${ }^{1,2,95,96}$ We map the effective tensor index $\nu$ to combinations of indices of rank-3 and rank-4 tensors as listed in Tab. II, and remap the index $\phi=\{x, y, z\} \rightarrow\{1,2,3\}$. Moreover, the summation over $\omega_{s}$ includes the combinations of all external frequencies that generate the same output frequency. As an example, consider the element 14 of the effective tensor for the OR process (that requires the combination of spatial indices and different frequency components)

$$
\begin{aligned}
\bar{\sigma}_{14}^{(2)}(0) & \equiv \sigma_{x x y}^{(2)}(\omega,-\omega)+\sigma_{x x y}^{(2)}(-\omega, \omega) \\
& +\sigma_{x y x}^{(2)}(\omega,-\omega)+\sigma_{x y x}^{(2)}(-\omega, \omega) .
\end{aligned}
$$

In Sec. A 3, we show that all spurious divergences present in the direct evaluation of the nonlinear conductivity tensors $\sigma_{\phi \lambda \ldots \alpha}^{(n)}\left(\omega_{n}, \ldots, \omega_{1}\right)$ vanish when considering the relevant effective rank-2 tensor $\bar{\sigma}_{\phi \nu}^{(n)}\left(\omega_{s}\right)$, for secondand third-order processes, namely Eqs. (A5) and (A6). In addition, we identify the remaining physical divergences
TABLE II. Mapping of index $\nu$ to combinations of Cartesian indices for the second-order $(\lambda \alpha)$ and third-order $(\lambda \beta \alpha)$ responses, respectively.

\begin{tabular}{lcc}
\hline \hline$\nu$ & $\lambda \alpha$ & $\lambda \beta \alpha$ \\
\hline 1 & $x x$ & $x x x$ \\
2 & $y y$ & $y y y$ \\
3 & $z z$ & $z z z$ \\
4 & $x y+y x$ & $y z z+z y z+z z y$ \\
5 & $y z+z y$ & $y y z+y z y+z y y$ \\
6 & $z x+x z$ & $z z x+z x z+x z z$ \\
7 & & $z x x+x z x+x x z$ \\
8 & & $x y y+y x y+y y x$ \\
9 & & $x x y+x y x+y x x$ \\
0 & & $x y z+x z y+y z z+y x z+z x y+z y x$ \\
\hline \hline
\end{tabular}

occurring at zero frequency. The origin of these divergences can be traced to the diagonal elements of the density matrix, i.e. $\rho_{m m}^{(n)}$. The linear order the density matrix $\rho_{m m}^{(1)}$ introduces a divergent term in the linear optical response

$$
\sigma_{\phi \alpha}^{i}(\omega)=-\frac{2 i g \sigma_{1}}{\Omega} \frac{1}{\omega+i \eta} \sum_{\mathbf{k}} \sum_{m} v_{m m}^{\phi} \frac{\partial f_{m}}{\partial k_{\alpha}} .
$$

By keeping the adiabatic parameter finite, rather than taking the formal adiabatic limit $\eta \rightarrow 0^{+}$, the response remains finite and captures the so-called Drude term, ${ }^{97-99}$ with the finite $\eta$ representing the scattering rate. Similar divergences are found in terms beyond the linear order, defining nonlinear Drude-like processes. The quadratic response contains two such terms, a linear divergence in Eq. (A10) and a quadratic in Eq. (A11), whereas the cubic response spawns a total of four terms that define a linear and a cubic divergence. The cubic term emerges from Eq. (A4d) and the linear divergence stems from Eqs. (A14), (A15b), and (A17). By the same token, $\eta$ should be kept finite and mapped to the scattering rate of each nonlinear Drude-like process. To avoid confusion with the spurious divergences, we refer to these as nonlinear Drude-like terms. Spurious divergences are found in almost all contributions that involve intraband processes, with the exception of the purely intraband processes and also those comprised by a single interband transition $(e)$ followed by multiple intraband processes $(i)$, i.e. processes labeled as $i e$, iie, and so forth. In contrast to the intensive study of the linear optical response of weakly disordered MLG, ${ }^{70-74}$ little progress has been made on the characterization of the nonlinear Drude terms. To qualitatively identify all contributions from the Drude-like terms, we consider a simple model based on a fixed scattering rate approximation. The choice of such method restricts the characterization to pristine or weakly disordered systems, as in disordered systems the scattering rates are dependent on doping level. ${ }^{100}$ In the absence of a proper estimate for the nonlinear scattering 
rates, ${ }^{30}$ we take the rate for the linear process in weakly disordered MLG as a reference figure for all scattering rates, thereby setting $\hbar \eta=10 \mathrm{meV}$ for two reasons. First, it represents the finite scattering rate of charge carries in the system, used successfully in the analysis of the linear response of graphene in the presence of weak disorder. ${ }^{70-74}$ Second, it facilitates the convergence of the calculation over the entire spectrum, particularly for the contributions associated with interband transitions.

The lattice symmetry determines the number of independent and finite elements in the optical conductivity and susceptibility tensors, which in turn determine the properties of the respective effective tensors. Restricting the external fields to normal incidence limits the response to the in-plane motion of electrons in the crystal. For threefold symmetric crystals, such as graphene-based systems, the third-order effective tensor contains only one independent tensor element $\bar{\sigma}_{11}^{(3)}=\bar{\sigma}_{18}^{(3)}=\bar{\sigma}_{29}^{(3)}=\bar{\sigma}_{22}^{(3)}$. Breaking the inversion center of the honeycomb lattices, while preserving the threefold symmetry, allows for second-order effects, e.g. SHG and OR, that are governed by $\bar{\sigma}_{14}^{(2)} / 2=\bar{\sigma}_{21}^{(2)}=-\bar{\sigma}_{22}^{(2)}$.

\section{RESULTS}

Throughout this section we address several linear and nonlinear optical response functions of MLG and BBG. Although the linear optical conductivity has been studied intensively, ${ }^{70-74}$ we briefly discuss it here to serve as a basis for our analysis of the nonlinear response. We restrict our analysis to nonlinear interactions with a monochromatic field, namely THG $\bar{\sigma}_{\phi \nu}^{(3)}\left(\omega_{s}=3 \omega\right)$, optical Kerr conductivity $\bar{\sigma}_{\phi \nu}^{(3)}\left(\omega_{s}=\omega\right)$, and SHG $\bar{\sigma}_{\phi \nu}^{(2)}\left(\omega_{s}=2 \omega\right)$. For THG in graphene we show that our spectra are in agreement with previous results computed within the Dirac approximation..$^{28-30,77,78}$

\section{A. Optical conductivity}

The effect of doping on the linear optical response of MLG has been discussed extensively. ${ }^{70-74}$ It manifests itself as a combination of Pauli blocking and a Drude low-frequency peak. Pauli blocking suppresses the interband optical response below the chemical potential $|\mu|$, i.e. $\hbar \omega<2|\mu|$, as shown in Fig. 2a, while the intraband motion [governed by the Drude peak, Eq. (9)] is characterized by the finite scattering rate of charge carriers. Note that we only consider $n$-doping, i.e. $\mu>0$. In the low-energy regime, the energy dispersions of MLG and BBG are nearly electron-hole symmetric, even considering next-nearest neighbors hopping in the Hamiltonian. Hence, $p$-doping of an equal magnitude would lead to essentially identical optical response. A doping level $\mu=397 \mathrm{meV}$ sets the Pauli blocking threshold at $\hbar \omega=2 \mu=793 \mathrm{meV}$, corresponding to the reference

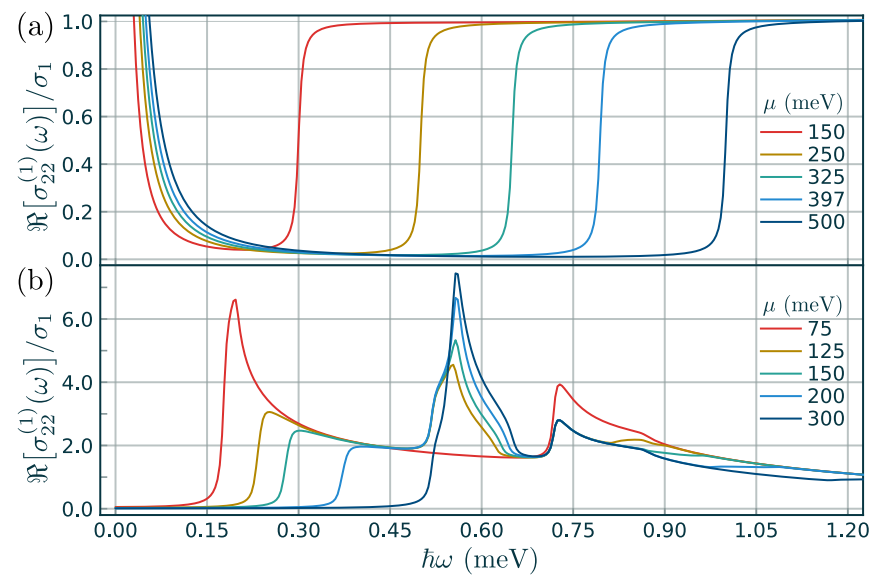

FIG. 2. Linear optical conductivity (in units of $\sigma_{1}=e^{2} / 4 \hbar$ ) for MLG (a) and BBG (b) as a function of energy for several $\mu$ taking $T=1 \mathrm{~K}$ and $\Delta=200 \mathrm{meV}$.

wavelength $\lambda \sim 1560 \mathrm{~nm} \cdot{ }^{101,102}$ The response of BBG in Fig. $2 \mathrm{~b}$ is significantly richer than that of the monolayer. Manifestations of Pauli blocking are still present, but the tuneable chemical potential enables and disables several transitions associated with the gap (for BBG) and the interlayer hopping $\gamma_{1}$. In contrast to the featureless response of MLG, the transitions associated with the lowenergy vHs introduce several resonances that dominate the optical response of BBG. In Fig. 2b, we show the linear response of BBG $(\Delta=200 \mathrm{meV})$ as a function of photon energy for several $\mu$. Unlike MLG, BBG supports a rich optical response that is highly sensitive to doping, e.g. the large and tuneable resonance that emerges in the vicinity of $\hbar \omega \sim 0.6 \mathrm{eV}$, whenever doping is large enough to populate the first conduction band with electrons or to introduce holes in the top valence band that provide an additional set of allowed resonant transitions.

\section{B. Nonlinear response of MLG}

Regarding the nonlinear response of graphene, it has been shown ${ }^{28-30,77,103}$ that the THG can be strongly enhanced and tuned by controlling the doping via electrostatic gating. Our calculations and those of Refs. 28 and 30 start from the evaluation of the response function using the $\mathrm{LG},{ }^{26}$ but differ at several levels. Alternative approaches have been used in Refs. 29 and 78 and references therein, where the electric field is assumed to be space-dependent. More recently, non-perturbative methods have been used to compute the nonlinear response of graphene using the Dirac Hamiltonian. ${ }^{79}$

The present evaluation of the nonlinear response incorporates the effects of finite temperature from the onset, rather than estimating finite temperature conductivity from zero temperature calculations. ${ }^{77}$ It naturally extends beyond the particular solutions for the Dirac model, ${ }^{28-30,77,78}$ as none of the initial expressions used, 

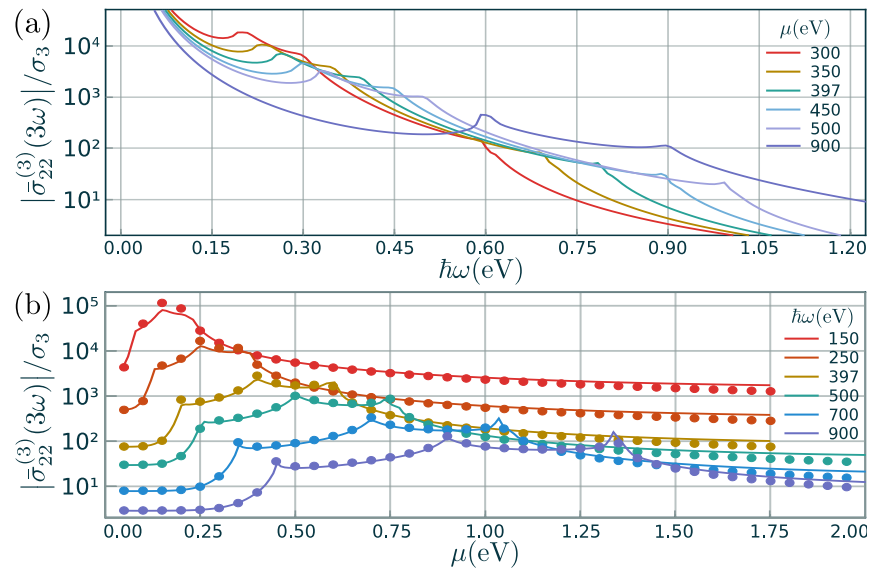

FIG. 3. Magnitude of THG conductivity of doped graphene in units of $\sigma_{3} \equiv e^{4} a_{0}^{2} /\left(8 \gamma_{0}^{2} \hbar\right)=6.28 \times 10^{-26} \mathrm{Sm}^{2} / \mathrm{V}^{2}$, computed from full dispersion TB using Eqs. (A6). In (a), we plot the frequency-dependent THG conductivity at several finite chemical potentials. (b) shows the dependence on the chemical potential for several photon frequencies, computed with full dispersion TB (solid lines) and Dirac approximation at zero temperature (dots) using Eq. (10).

Eqs. (A4), are derived for a specific Hamiltonian or fixed number of bands. Therefore, they are valid for more complex systems that require more elaborate Hamiltonians. Although the Dirac approximation has proven useful for the characterization of the low-energy linear- and thirdorder optical response of graphene, ${ }^{28-30,77,78,81}$ it is of little use for the study of any quadratic response, as all even-order processes vanish in the presence of full rotation symmetry, at least within the dipole approximation.

Temperature plays an important role in nonlinear optics. It may not only soften the resonant features present in the optical response by modifying the effective electron distribution, ${ }^{58,66,104,105}$ but also change the scattering rates. The leading order effects on the second- ${ }^{22,76}$ and third-order ${ }^{28,30}$ responses are the broadening of the resonant features. In addition, temperature can switch on transitions that would otherwise be forbidden due to Pauli blocking, but this is more frequently than not a minor effect, when compared with the strong resonances associated with large $\mu .{ }^{22,76}$ For the sake of brevity we refrain from discussing the effects of temperature in detail. Unless stated otherwise, results shown in this paper were computed for $T=10 \mathrm{~K}$, such that thermal broadening remains minimal and manifestations of Pauli blocking are preserved, thus allowing for a clear identification of all processes involved in the optical response. In realistic experimental scenarios, ${ }^{30}$ the effective carrier temperature lies in the range $T \sim 1000-1500 \mathrm{~K}$ and scattering rates are likely to be different.

\section{Third harmonic generation}

In Fig. 3a, we plot the magnitude of the THG conductivity $\bar{\sigma}_{22}^{(3)}(3 \omega)$ at finite doping, computed from full dispersion TB using Eqs. (A6). Similarly to the linear response, the Drude-like nonlinear response, comprised by Eqs. (A14), (A15b) and (A17), has a smooth featureless power law decay, that dominates the THG response below the first resonance associated with the chemical potential. In the present model, with a single nonlinear scattering rate, the magnitude of the Drude-like response at the DC limit and the broadening of the resonances associated with the Fermi level, i.e. $\hbar \omega / \mu=\{2 / 3,1,2\}$, are both controlled by the same parameter. Therefore, in the presence of sufficiently large scattering rates $\hbar \eta>50$ $\mathrm{meV}$ the resonances are strongly suppressed and the response reduces to the Drude-like contributions. For moderate nonlinear scattering rate, the regular part clearly surpasses the Drude-like terms in the vicinity of the resonances associated with the Fermi level. As in previous studies ${ }^{28-30,78,81}$ results show that the nonlinear response can be tuned by controlling $\mu$, as the resonances are shifted by the chemical potential. Temperature and the nonlinear scattering rate play a large role and can significantly soften the resonances. Moreover, it is worth noting that the resonance $\hbar \omega / \mu=2 / 3$ lies within the energy range of minimal absorption due to Pauli blocking. Also, this resonance should not be disturbed by the optical Kerr effect on absorption, as the latter can only generate resonances at $\hbar \omega / \mu=\{1,2\}$ as discussed in detail below.

The general solutions for the third-order response, Eqs. (A6), can be integrated analytically for THG by taking the zero temperature limit and making use of the Dirac dispersion

$$
\begin{aligned}
\frac{\bar{\sigma}_{22}^{(3)}}{\sigma_{3}} & =\frac{-3 i}{1024 \pi} \frac{\gamma_{0}^{4}}{(\hbar \bar{\omega})^{4}}\left[45 \log \left(\frac{2 \mu-3 \hbar \bar{\omega}}{2 \mu+3 \hbar \bar{\omega}}\right)\right. \\
& \left.-64 \log \left(\frac{2 \mu-2 \hbar \bar{\omega}}{2 \mu+2 \hbar \bar{\omega}}\right)+17 \log \left(\frac{2 \mu-\hbar \bar{\omega}}{2 \mu+\hbar \bar{\omega}}\right)\right],
\end{aligned}
$$

in agreement with previous results. ${ }^{28}$ Likewise the divergent terms present in THG can be determined straightforwardly from the general solutions for the Drude-like terms. The linear divergence stems from Eqs. (A15b) and (A17)

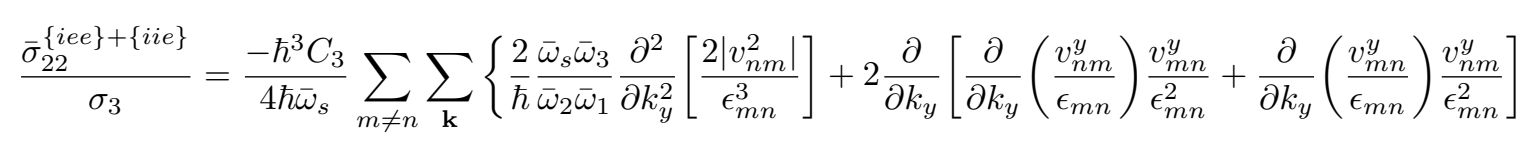




$$
\left.+\frac{\left(\bar{\omega}_{2}+\bar{\omega}_{1}\right)^{2}}{\bar{\omega}_{2} \bar{\omega}_{1}} \frac{\partial}{\partial k_{y}}\left[\frac{v_{n n}^{y}-v_{m m}^{y}}{\epsilon_{m n}^{2}} \frac{2\left|v_{n m}^{y}\right|^{2}}{\epsilon_{m n}^{2}}\right]+\frac{\partial\left(v_{n n}^{y}-v_{m m}^{y}\right)}{\partial k_{y}} \frac{2\left|v_{n m}^{2}\right|}{\epsilon_{m n}^{4}}\right\} f_{n m} \simeq \frac{45 i \gamma_{0}^{4}}{256 \pi \mu^{3} \hbar \bar{\omega}}
$$

and the cubic term follows from Eq. (A13)

$$
\frac{\bar{\sigma}_{22}^{i i i}}{\sigma_{3}}=\frac{\hbar C_{3}}{\hbar^{3} \bar{\omega}_{s}\left(\bar{\omega}_{2}+\bar{\omega}_{1}\right) \bar{\omega}_{1}} \sum_{n} \sum_{\mathbf{k}} \frac{\partial^{3} v_{n n}^{y}}{\partial k_{y}^{3}} f_{n} \simeq \frac{9 i \gamma_{0}^{4}}{128 \pi \mu(\hbar \bar{\omega})^{3}} .
$$

In VG, spurious divergences appear for gapped systems if a truncated band structure is applied. ${ }^{24}$ For gapless twoband systems neither LG nor VG exhibit spurious divergences in the DC limit. Therefore, the Drude-like terms can always be derived from the full non-regularized expressions Eqs. (A4) by means of a Taylor series. Eqs. (11) are in agreement with the Taylor expansion of Eq. (10), which reads

$$
\begin{aligned}
\frac{\bar{\sigma}_{22}^{(3)}}{\sigma_{3}} & =\frac{9 i \gamma_{0}^{4}}{128 \pi \mu(\hbar \bar{\omega})^{3}}+\frac{45 i \gamma_{0}^{4}}{256 \pi \mu^{3} \hbar \bar{\omega}} \\
& +\frac{3339 i \gamma_{0}^{4} \hbar \bar{\omega}}{10240 \pi \mu^{5}}+\mathcal{O}\left(\bar{\omega}^{3}\right) .
\end{aligned}
$$

The divergence with doping $\mu$ is a mere artifact of the Taylor series computed in the limit $\hbar \omega \ll \mu$. This becomes evident by considering opposite limit $\mu \ll \hbar \omega$

$$
\frac{\bar{\sigma}_{22}^{(3)}}{\sigma_{3}}=\frac{3 \gamma_{0}^{4}}{512(\hbar \bar{\omega})^{4}}-\frac{i \gamma_{0}^{4} \mu^{3}}{6 \pi(\hbar \bar{\omega})^{7}}+\mathcal{O}\left(\mu^{4}\right)
$$

Our result not only offers a clear separation between the regular and divergent terms, it also offers a general model for the nonlinear Drude-like terms, that can be used to characterize the nonlinear scattering rate beyond the constraints of the Dirac approximation. In Fig. 3b, we show the dependence of the THG response in MLG as a function of doping level, evaluated using two different models, namely: (i) full TB dispersions using Eqs. (A6); (ii) the analytic result derived using the Dirac approximation at zero temperature Eq. (10). The agreement between the exact solution and the numerically integrated expressions Eqs. (A6) is remarkable, even for large doping levels $\mu \sim 1.5 \mathrm{eV}$, indicating that the closed form expressions in Eq. (10) can be used to accurately characterize the THG response of graphene, even in cases of large photon energy and substantial doping. The agreement between the exact solution and the numerically integrated expression extends to the Drude-like features.

Data shown in Fig. 3 highlight the strongly varying nature of the nonlinear response in graphene with respect to the photon energy. We present "figure of merit" estimates for the nonlinear susceptibility based on the finite temperature $T=10 \mathrm{~K}$ results. Additional enhancement of the magnitude of the response can be achieved by considering smaller nonlinear scattering rates. The 3D nonlinear susceptibility is evaluated from the 2D nonlinear conductivity using $\left|\chi^{(3)}\left(\omega_{s}\right)\right|=\left|\sigma^{(3)}\left(\omega_{s}\right)\right| /\left(c_{0} \varepsilon_{0} \omega_{s}\right)$,

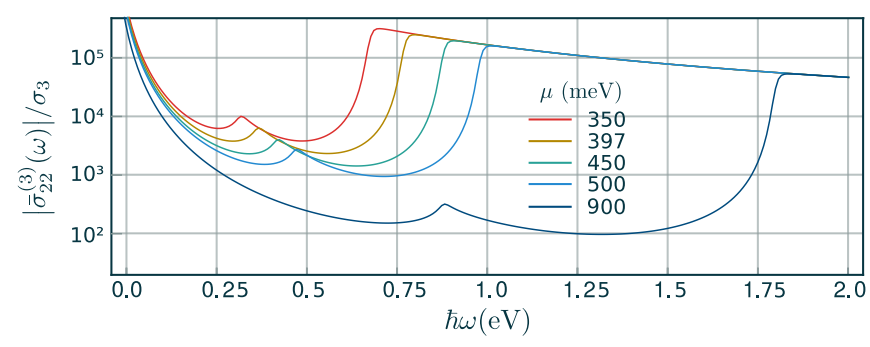

FIG. 4. Frequency dependence of the optical Kerr conductivity conductivity of MLG in units of $\sigma_{3}$ for several chemical potentials $\mu$.

with $\varepsilon_{0}$ being the vacuum permittivity, and $c_{0}=3.35$ $\AA$ the interplanar distance in graphite. ${ }^{86}$ Given the absence of an accurate estimate for the nonlinear scattering rate, we refrain from showing data obtained in the region where the Drude-like terms dominate. Considering the main THG resonances at $\mu=\{300,900\}$ $\mathrm{meV}$, i.e. $\hbar \omega \sim\{200,600\} \mathrm{meV}$, the response can be tuned up to $\left|\chi^{(3)}(3 \omega)\right|=\{504,4.2\} \times 10^{-18} \mathrm{~m}^{2} / \mathrm{V}^{2}$. Our estimates provide figures comparable with some previous experimental reports for THG in graphene that range from $\sim 10^{-19}$ to $\sim 10^{-15} \mathrm{~m}^{2} / \mathrm{V}^{2} \cdot 4,12,101,106 \mathrm{It}$ is worth noting that the THG susceptibility of doped graphene can significantly exceed that of other materials, e.g. gold ${ }^{107}\left|\chi^{(3)}\right| \sim 10^{-19} \mathrm{~m}^{2} / \mathrm{V}^{2}$ and AlGaAs ${ }^{108,109}$ $\left|\chi^{(3)}\right| \sim 10^{-18} \mathrm{~m}^{2} / \mathrm{V}^{2}$.

\section{Optical Kerr effect}

In addition to the $n^{\text {th }}$ harmonic generation, the interaction of an intense monochromatic electromagnetic field with a crystal also generates nonlinearities associated with combinations of positive and negative frequency components of the field. At third-order, it generates a nonlinear current density with the frequency of the driving field. This optical Kerr conductivity $\bar{\sigma}_{22}^{(3)}(\omega)$ gives rise to intensity-dependent effects on the refractive index. ${ }^{1,2}$ Following the analysis of the THG, we show results for the optical Kerr conductivity $\left|\sigma^{(3)}(\omega)\right|$ in Fig. 4. As expected from the expression for the third-order conductivity tensor, Eqs. (A4), this response contains two resonances, $\hbar \omega=2 \mu\{1 / 2,1\}$. The former stems from the two photon resonance $\propto 1 /\left(\bar{\omega}_{2}+\bar{\omega}_{1}-\epsilon_{m n} / \hbar\right)$, whenever $\omega_{2}=\omega_{1}$. The latter originates from the single and three 
photon resonances. All terms involving the three photon resonance $\propto 1 /\left(\bar{\omega}_{3}+\bar{\omega}_{2}+\bar{\omega}_{1}-\epsilon_{m n} / \hbar\right)$ reduce to a single photon resonance due to the presence of frequencies with opposite sign.

The contrast between portions of the spectra dominated by Drude-like and regular parts of the optical Kerr conductivity is stronger than in the THG response. Even at moderate doping levels, $\mu \sim 200 \mathrm{meV}$, contributions from the regular part to the first resonance $\hbar \omega=\mu$, clearly surpass the Drude-like peak. Moreover, from the onset of the second resonance $\hbar \omega=2 \mu$, the regular part of the response exhibits a strong steplike feature and becomes the leading term driving the response. The Drude-like terms show little dependence on the details of the band structure and, similarly, the Fermi level only affects the overall magnitude of the Drude response but not the shape. The robust increase of the response at the second resonance $\hbar \omega=2 \mu$, indicates that the dependence of the optical Kerr effect on doping could be experimentally probed in highly doped graphene samples, $\mu \sim 397 \mathrm{meV}$. For such samples, the $\hbar \omega=2 \mu$ resonance should lie within range of high intensity lasers frequently used for nonlinear optics experiments $\hbar \omega \sim 793 \mathrm{meV}$, i.e. $\lambda \sim 1560 \mathrm{~nm} .{ }^{101,102}$ It is worth noting that the low-energy results are in agreement with previous estimates using the Dirac model. ${ }^{28,29,78}$ Using data from Fig. 4b, the magnitude the optical Kerr susceptibility at $\mu=350 \mathrm{meV}$ for $\hbar \omega=\{350,700\} \mathrm{meV}$ reads $\left|\chi^{(3)}(\omega)\right| \sim\{43,4.3\} \times 10^{-15} \mathrm{~m}^{2} / \mathrm{V}^{2}$, indicating significantly larger third-order susceptibilities than those observed in THG.

\section{Response of gapped graphene}

Below, we consider the nonlinear response in noncentrosymmetric systems, such as commensurate structures of graphene on hBN substrates, where the electronic dispersion of graphene is gapped.

For doped systems, the contributions to the third-order response that emerge from features associated with the gap, $E_{g} \sim 30 \mathrm{meV}$, are dwarfed by the Drude-like terms. Moreover, in the case of large doping $\mu \gg E_{g}$, the interband transitions are strongly suppressed by Pauli blocking, while the features associated with resonances at the Fermi level nearly match the resonances found in the gapless dispersion. Therefore, we only consider the implications of symmetry breaking on the quadratic response.

In previous studies, ${ }^{35,76}$ the authors addressed the quadratic response of non-centrosymmetric honeycomb lattices in the regime where transitions between the top valence and bottom conduction bands dominate. Here, we focus on the regime where the chemical potential is significantly larger than the band gap $\mu \gg E_{g}$. Considering typical values for the Fermi level in graphene $\mu \sim 250$ $\mathrm{meV}$, it is feasible to reach this regime in systems such as graphene on hBN with $E_{g} \sim 31 \mathrm{meV} .{ }^{34}$ The Fermi level suppresses the resonances associated with the band gap,

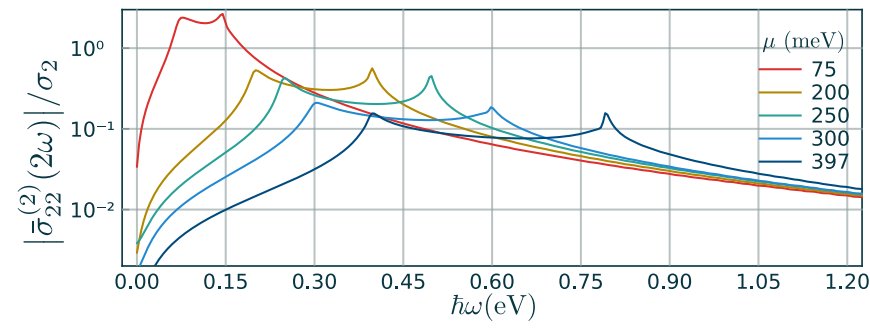

FIG. 5. SHG conductivities $\bar{\sigma}_{22}^{(2)}(2 \omega)$ of gapped graphene in units of $\sigma_{2} \equiv e^{3} a_{0} /\left(4\left|\gamma_{0}\right| \hbar\right)=2.88 \times 10^{-15} \mathrm{Sm} / \mathrm{V}$ with $E_{g}=$ $\delta=30 \mathrm{meV}$.

$\hbar \omega \sim E_{g}\{1 / 2,1\}$ and the response is then controlled by the mixed inter-intraband processes occurring at the energy scale of the Fermi level $\hbar \omega=\{\mu, 2 \mu\}$. Figure 5 shows the magnitude of the SHG conductivity for several $\mu$ as a function of photon energy, indicating that the response is essentially confined to resonances with energy associated with the Fermi level. Moreover, for sufficiently large doping levels, $\mu \sim 150 \mathrm{meV}$, the lowest energy resonance in the SH spectrum is sufficiently energetic to avoid the large absorption associated with the Drude peak, i.e. it remains within the Pauli blocked region of the spectrum. The magnitude of the SH features at high doping is significantly smaller than for weak doping. Nonetheless, it remains comparable to the estimates of SHG in BBG. ${ }^{35}$ Therefore, SHG should be detectable in commensurate structures of graphene on $\mathrm{SiC}$ or $\mathrm{hBN}$, and also tuneable by doping. Similar results are found for the OR process, where the magnitude of the response at large doping is comparable to that of BBG in the same energy range. ${ }^{76}$

It is worth noting that, in systems with identical occupation of the $\mathbf{K}$ and $\mathbf{K}^{\prime}$ valleys, the quadratic response emerges solely from the mixed ie and purely interband $e e$ processes, as the remaining processes, $e i$ and $i i$, cancel out upon integration over the full Brillouin zone. Yet, in systems out-of-equilibrium such as valley polarized honeycomb lattices, the $e i$ and $i i$ processes no longer vanish ${ }^{22}$ and may enhance the response even further.

\section{Nonlinear response of BBG}

As discussed in the context of the linear response, the presence of several $\mathrm{vHs}$ in the low-energy dispersion of BBG introduces additional resonances. The presence of broadening softens these features, converting otherwise resonances to small bumps, cf. Fig. 6a. In addition, it also contains resonances that stem from the intraband motion at the Fermi level. Considering doped BBG, $\mu=150 \mathrm{meV}$ and $\Delta=200 \mathrm{meV}$, the resonances at lowest energy $\hbar \omega \sim\{91,142\} \mathrm{meV}$ arise mainly from transitions associated with the Fermi level. In contrast with the THG response, the resonant features present in the optical Kerr effect are robust and significantly larger than the contributions from the Drude-like terms, Fig. 6 b. The features arise from transitions occurring at 


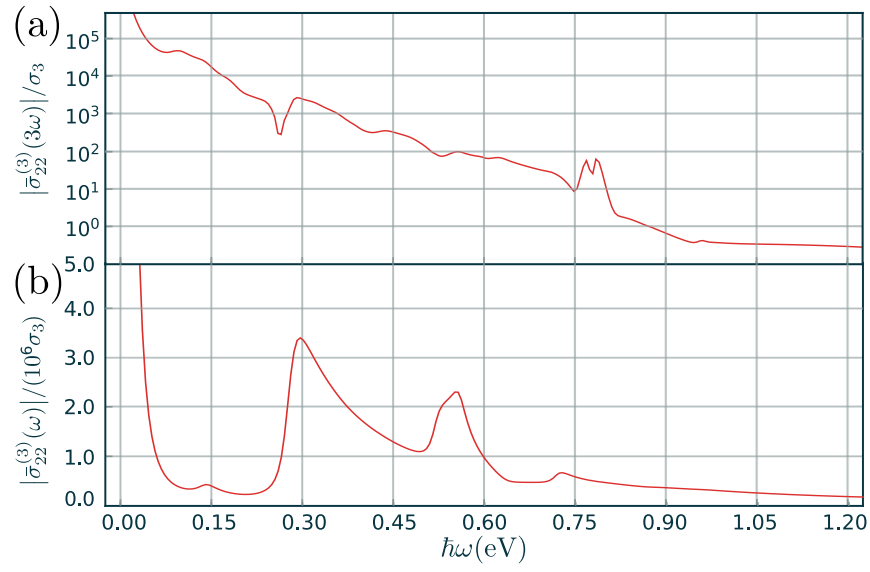

FIG. 6. Third-order response of BBG (in units of $\sigma_{3}$ ) with $\Delta=200 \mathrm{meV}, \mu=150 \mathrm{meV}$, showing (a) the THG response and (b) the optical Kerr conductivity.

the low-energy vHs and intraband motion at the Fermi level. The lowest energy resonances that stem from intraband motion are found at $\hbar \omega \sim\{137,274\} \mathrm{meV}$, while the dominant resonance at $\hbar \omega \sim 300 \mathrm{meV}$ arises from the doping cut-off of the vHs resonances that are located at $\hbar \omega \sim\left\{\delta_{a}^{2}, \delta_{b}^{2}, \delta_{c}^{2}\right\} / 2 \sim\{293,267,259\} \mathrm{meV}$. The results shown in Fig. 6b indicate that the largest resonance stems from the combination of both processes, yet it is important to note that this combination is accidental, as the energies of the resonances depend on the details of the band structure and, also, on the Fermi level.

The amplitude of the THG and optical Kerr conductivities in BBG is significantly larger than in MLG. The THG nonlinear susceptibility at the lowest resonances associated with doping and the vHs, $\hbar \omega \sim\{91,274\} \mathrm{meV}$, read $\left|\chi^{(3)}(3 \omega)\right| \sim\{202,9.1\} \times 10^{-16} \mathrm{~m}^{2} / \mathrm{V}^{2}$, respectively. Regarding the optical Kerr effect in BBG the amplitude of the most intense resonance $\hbar \omega \sim 274 \mathrm{meV}$ reads $\left|\chi^{(3)}(\omega)\right| \sim 1.7 \times 10^{-13} \mathrm{~m}^{2} / \mathrm{V}^{2}$. Akin to the results for MLG, doping plays a crucial role as the resonances associated with the intraband motion can be displaced and, furthermore, it can suppress the resonances associated with the low energy vHs of BBG.

\section{CONCLUDING REMARKS}

We have derived length gauge optical response functions up to third-order for a general periodic system. The expressions for the effective nonlinear conductivity tensors are not only valid for any periodic system at finite temperature and doping, but are also free of any spurious divergences and all Drude-like terms are identified. The spurious divergences are proven to vanish by considering effective rank-2 tensors, which comprise all contributions to the relevant physical observable, as introduced in Sec. II C and explicitly shown in the appendix. We identify all terms that contribute to the nonlinear Drude- like response in the DC limit, providing the basis for a comprehensive study of the scattering rates in nonlinear processes in $2 \mathrm{D}$ materials.

The expressions for the effective conductivity tensors are then used to evaluate the optical response of doped MLG and BBG. We compute the optical conductivity and several nonlinear response functions, namely THG, optical Kerr effect, and SHG. All results are strongly dependent on doping, showing that the nonlinear thirdorder susceptibility can be tuned over several orders of magnitude, in line with experimental reports for THG in graphene. ${ }^{4,12,101,106}$ By comparing the exact solutions derived with the Dirac approximation, with numerical integration using full dispersion TB models, our results show that the Dirac approximation provides remarkably accurate results, for THG in MLG even at very large doping. The results show that the third-order response can be highly dependent on contributions that arise from nonlinear Drude-like terms, stressing the necessity for further studies probing the nonlinear optical response in the low-frequency regime, and also more elaborate theoretical models that can describe accurately scattering in nonlinear processes.

Proceeding beyond pristine MLG, we show that the second-order response of systems with small gaps can be strongly enhanced by the presence of finite doping, especially in the regime where the chemical potential is significantly larger than the energy gap. The nonlinear response of BBG exhibits many resonant features that arise from two sources: transitions occurring at the vHs in the vicinity of the Dirac points, present even in charge neutral systems, and the contributions associated with intraband motion occurring at the Fermi level. This leads to richer nonlinear conductivity spectra that deviate strongly from the simple and well-localized resonances in MLG occurring at $\hbar \omega / \mu=\{2 / 3,1,2\} .28,30,78$

\section{ACKNOWLEDGMENTS}

This work was supported by the QUSCOPE center sponsored by the Villum Foundation, and TGP is supported by the CNG center under the Danish National Research Foundation, project DNRF103.

\section{Appendix A: Length gauge response}

In this appendix, we present all relevant results for the evaluation of the current density response. We start by listing the elements of the power series expansion of the density matrix $\rho_{m n}(t)$ in the electromagnetic field, up to third-order, followed by the respective conductivity tensors elements. We then proceed with a detailed description of the procedure used to isolate all the divergences present in the expressions for the conductivity tensor, terminating with the separation of the physical 
Drude-like terms from the spurious divergences that are proven to vanish.

The power series solution of the quantum Liouville equation is determined using the procedure outlined in
Refs. 26 and 76. Using the notation defined in Ref. 76, the perturbative expansion of the density matrix up to third-order reads

$$
\begin{aligned}
& \rho_{m n}^{(1)}(t) \equiv \rho_{m n}^{e}(t)+\rho_{m n}^{i}(t)=\frac{e}{2 \hbar} \sum_{\omega_{1}}\left[\bar{\delta}_{m n} \mathcal{A}_{m n}^{\alpha} f_{n m}-i \delta_{m n} \frac{\partial f_{n}}{\partial k_{\alpha}}\right] \frac{E_{\omega_{1}}^{\alpha} e^{-i \bar{\omega}_{1} t}}{\bar{\omega}_{1}-\omega_{m n}}, \\
& \rho_{m n}^{(2)}(t) \equiv \rho_{m n}^{e e}(t)+\rho_{m n}^{e i}(t)+\rho_{m n}^{i e}(t)+\rho_{m n}^{i i}(t) \\
& =\frac{e^{2}}{4 \hbar^{2}} \sum_{\omega_{2} \omega_{1}} \sum_{l} \frac{E_{\omega_{2}}^{\lambda} E_{\omega_{2}}^{\alpha} e^{-i\left(\bar{\omega}_{2}+\bar{\omega}_{1}\right) t}}{\bar{\omega}_{2}+\bar{\omega}_{1}-\omega_{m n}}\left[\bar{\delta}_{l m} \bar{\delta}_{l n}\left(\frac{\mathcal{A}_{m l}^{\lambda} \mathcal{A}_{l n}^{\alpha} f_{n l}}{\bar{\omega}_{1}-\omega_{l n}}-\frac{f_{l m} \mathcal{A}_{m l}^{\alpha} \mathcal{A}_{l n}^{\lambda}}{\bar{\omega}_{1}-\omega_{m l}}\right)-i \delta_{l m} \bar{\delta}_{m n} \frac{\mathcal{A}_{m n}}{\bar{\omega}_{1}} \frac{\partial f_{n m}}{\partial k_{\alpha}}\right. \\
& \left.-i \delta_{l m} \bar{\delta}_{m n}\left(\frac{\mathcal{A}_{m n}^{\alpha} f_{n m}}{\bar{\omega}_{1}-\omega_{m n}}\right)_{; \lambda}-\frac{\delta_{l m} \delta_{m n}}{\bar{\omega}_{1}} \frac{\partial^{2} f_{n}}{\partial k_{\lambda} \partial k_{\alpha}}\right] \\
& \rho_{m n}^{(3)}(t) \equiv \rho_{m n}^{e e e}(t)+\rho_{m n}^{e i e}(t)+\rho_{m n}^{e e i}(t)+\rho_{m n}^{e i i}(t)+\rho_{m n}^{i e e}(t)+\rho_{m n}^{i i e}(t)+\rho_{m n}^{i e i}(t)+\rho_{m n}^{i i i}(t) \\
& =\frac{-e^{3}}{8 \hbar^{3}} \sum_{\omega_{3} \omega_{2} \omega_{1}} \frac{E_{\omega_{3}}^{\lambda} E_{\omega_{2}}^{\beta} E_{\omega_{1}}^{\alpha} e^{-i \bar{\omega}_{s} t}}{\bar{\omega}_{s}-\omega_{m n}}\left\{\sum _ { p l } \left[\frac{\mathcal{A}_{m p}^{\lambda} \bar{\delta}_{m p} \bar{\delta}_{l p} \bar{\delta}_{l n}}{\bar{\omega}_{2}+\bar{\omega}_{1}-\omega_{p n}}\left(\frac{\mathcal{A}_{p l}^{\beta} \mathcal{A}_{l n}^{\alpha} f_{n l}}{\bar{\omega}_{1}-\omega_{l n}}-\frac{f_{l p} \mathcal{A}_{p l}^{\alpha} \mathcal{A}_{l n}^{\beta}}{\bar{\omega}_{1}-\omega_{p l}}\right)\right.\right. \\
& \left.-\frac{\bar{\delta}_{l m} \bar{\delta}_{l p} \bar{\delta}_{p n} \mathcal{A}_{p n}^{\lambda}}{\bar{\omega}_{2}+\bar{\omega}_{1}-\omega_{m p}}\left(\frac{\mathcal{A}_{m l}^{\beta} \mathcal{A}_{l p}^{\alpha} f_{p l}}{\bar{\omega}_{1}-\omega_{l p}}-\frac{f_{l m} \mathcal{A}_{m l}^{\alpha} \mathcal{A}_{l p}^{\beta}}{\bar{\omega}_{1}-\omega_{m l}}\right)\right] \\
& +i \sum_{l} \bar{\delta}_{m l} \bar{\delta}_{l n}\left[\frac{\mathcal{A}_{m l}^{\lambda}}{\bar{\omega}_{2}+\bar{\omega}_{1}-\omega_{l n}}\left(\frac{\mathcal{A}_{l n}^{\alpha} f_{n l}}{\bar{\omega}_{1}-\omega_{l n}}\right)_{; \beta}-\frac{\mathcal{A}_{l n}^{\lambda}}{\bar{\omega}_{2}+\bar{\omega}_{1}-\omega_{m l}}\left(\frac{\mathcal{A}_{m l}^{\alpha} f_{l m}}{\bar{\omega}_{1}-\omega_{m l}}\right)_{; \beta}\right] \\
& +i \sum_{l} \bar{\delta}_{m l} \bar{\delta}_{l n}\left[\frac{\mathcal{A}_{m l}^{\lambda} \mathcal{A}_{l n}^{\beta} / \bar{\omega}_{1}}{\bar{\omega}_{2}+\bar{\omega}_{1}-\omega_{l n}} \frac{\partial f_{n l}}{\partial k_{\alpha}}-\frac{\mathcal{A}_{m l}^{\beta} \mathcal{A}_{l n}^{\lambda} / \bar{\omega}_{1}}{\bar{\omega}_{2}+\bar{\omega}_{1}-\omega_{m l}} \frac{\partial f_{l m}}{\partial k_{\alpha}}\right]-\frac{\bar{\delta}_{m n} \mathcal{A}_{m n}^{\lambda} / \bar{\omega}_{1}}{\bar{\omega}_{2}+\bar{\omega}_{1}} \frac{\partial^{2} f_{n m}}{\partial k_{\beta} \partial k_{\alpha}} \\
& -i \sum_{l}\left(\frac{\bar{\delta}_{m l} \bar{\delta}_{l n}}{\bar{\omega}_{2}+\bar{\omega}_{1}-\omega_{m n}}\left(\frac{\mathcal{A}_{m l}^{\beta} \mathcal{A}_{l n}^{\alpha} f_{n l}}{\bar{\omega}_{1}-\omega_{l n}}-\frac{f_{l m} \mathcal{A}_{m l}^{\alpha} \mathcal{A}_{l n}^{\beta}}{\bar{\omega}_{1}-\omega_{m l}}\right)\right)_{; \lambda} \\
& \left.-\left(\frac{\bar{\delta}_{m n}}{\bar{\omega}_{2}+\bar{\omega}_{1}-\omega_{m n}}\left(\frac{\mathcal{A}_{m n}^{\alpha} f_{n m}}{\bar{\omega}_{1}-\omega_{m n}}\right)_{; \beta}\right)_{; \lambda}-\left(\frac{\bar{\delta}_{m n} \mathcal{A}_{m n}^{\beta} / \bar{\omega}_{1}}{\bar{\omega}_{2}+\bar{\omega}_{1}-\omega_{m n}} \frac{\partial f_{n m}}{\partial k_{\alpha}}\right)_{; \lambda}-i \frac{\delta_{m n} / \bar{\omega}_{1}}{\bar{\omega}_{2}+\bar{\omega}_{1}} \frac{\partial^{3} f_{n}}{\partial k_{\lambda} \partial k_{\beta} \partial k_{\alpha}}\right\},
\end{aligned}
$$

where $\omega_{s} \equiv \omega_{3}+\omega_{2}+\omega_{1}$ and $\omega_{m n}=\epsilon_{m n} / \hbar$. The perturbative expansion of the density matrix is then used to define the current density response as a power series in the electromagnetic field. From the current density, we define the linear and nonlinear conductivity tensors, namely: $\sigma_{\phi \alpha}^{(1)}$ the optical conductivity; $\sigma_{\phi \lambda \alpha}^{(2)}$ the quadratic response; $\sigma_{\phi \lambda \beta \alpha}^{(3)}$ the third-order response. The Fourier components of the optical conductivity tensor read

$$
\sigma_{\phi \alpha}^{(1)}\left(\omega_{1}\right) \equiv \sigma_{\phi \alpha}^{e}\left(\omega_{1}\right)+\sigma_{\phi \alpha}^{i}\left(\omega_{1}\right)=\frac{4 i g \sigma_{1}}{\Omega} \hbar^{2} \sum_{\mathbf{k}} \sum_{m n}\left(\frac{v_{n m}^{\phi} v_{m n}^{\alpha}}{\hbar \bar{\omega}_{1}-\epsilon_{m n}} \frac{\bar{\delta}_{m n} f_{n m}}{\epsilon_{m n}}-\frac{\delta_{m n}}{\hbar} \frac{v_{n n}^{\phi}}{\hbar \bar{\omega}_{1}} \frac{\partial f_{n}}{\partial k_{\alpha}}\right)
$$

where the conductivity scale is defined, for 2D systems, by the quantum of conductance $\sigma_{1}=e^{2} / 4 \hbar=\pi e^{2} / 2 h$ and the summation of all wave vectors $\mathbf{k}$ represents the integration over the entire Brillouin zone. In addition, the nondiagonal $(m \neq n)$ position matrix elements can be transformed to velocity matrix elements with $\mathcal{A}_{m n}^{\alpha} \equiv-i \hbar v_{m n}^{\alpha} / \epsilon_{m n}{ }^{26}$ To identify the inter- or intraband nature of the processes involved in any given tensor element, the interband processes are labeled by superscripts $e$, while the intraband motion is labeled by superscripts $i$. By the same token, we separate the contributions to quadratic response according to the nature of the two interactions. At second-order this spawns 4 processes: a purely interband $e e$ process, two mixed inter-intraband processes $e i$ and $i e$, and finally a purely intraband ii process. The respective Fourier components read

$$
\begin{aligned}
\sigma_{\phi \lambda \alpha}^{(2)}\left(\omega_{2}, \omega_{1}\right) & \equiv \sigma_{\phi \lambda \alpha}^{e e}\left(\omega_{2}, \omega_{1}\right)+\sigma_{\phi \lambda \alpha}^{e i}\left(\omega_{2}, \omega_{1}\right)+\sigma_{\phi \lambda \alpha}^{i e}\left(\omega_{2}, \omega_{1}\right)+\sigma_{\phi \lambda \alpha}^{i i}\left(\omega_{2}, \omega_{1}\right) \\
& =\frac{g \sigma_{2}}{\Omega} \frac{\hbar \gamma_{0}}{a} \sum_{\mathbf{k}} \sum_{m n} \frac{v_{n m}^{\phi}}{\hbar\left(\bar{\omega}_{2}+\bar{\omega}_{1}\right)-\epsilon_{m n}}\left[\hbar^{2} \sum_{l} \frac{\bar{\delta}_{l m} \bar{\delta}_{l n}}{\epsilon_{m l} \epsilon_{l n}}\left(\frac{v_{m l}^{\lambda} v_{l n}^{\alpha} f_{n l}}{\hbar \bar{\omega}_{1}-\epsilon_{l n}}-\frac{f_{l m} v_{m l}^{\alpha} v_{l n}^{\lambda}}{\hbar \bar{\omega}_{1}-\epsilon_{m l}}\right)\right.
\end{aligned}
$$




$$
\left.-\frac{\hbar \bar{\delta}_{m n}}{\hbar \bar{\omega}_{1}} \frac{v_{m n}^{\lambda}}{\epsilon_{m n}} \frac{\partial f_{n m}}{\partial k_{\alpha}}-\hbar \bar{\delta}_{m n}\left(\frac{v_{m n}^{\alpha} f_{n m} / \epsilon_{m n}}{\hbar \bar{\omega}_{1}-\epsilon_{m n}}\right)_{; \lambda}+\frac{\delta_{m n}}{\hbar \bar{\omega}_{1}} \frac{\partial^{2} f_{n}}{\partial k_{\lambda} \partial k_{\alpha}}\right]
$$

In contrast to linear response, the second-order conductivity scale, $\sigma_{2}$, depends explicitly on the physical properties of the system, namely the hopping energy $\gamma_{0}$ and the carbon-carbon bond length $a_{0}$. For the second-order conductivity of $2 \mathrm{D}$ systems, the scale is set by $\sigma_{2}=e^{3} a_{0} / 4\left|\gamma_{0}\right| \hbar$. At third-order, we obtain eight terms involving inter- and intraband processes, namely

$$
\begin{aligned}
& \sigma_{\phi \lambda \beta \alpha}^{e e e}\left(\omega_{3}, \omega_{2}, \omega_{1}\right)=-\frac{g \sigma_{3}}{i \Omega} \frac{\hbar^{4} \gamma_{0}^{2}}{a_{0}^{2}} \sum_{p l m n} \sum_{\mathbf{k}} \frac{\bar{\delta}_{m l} \bar{\delta}_{l p} \bar{\delta}_{p n}}{\epsilon_{m l} \epsilon_{l p} \epsilon_{p n}}\left[\frac{v_{n m}^{\phi}}{\hbar \bar{\omega}_{s}-\epsilon_{m n}}\left(\frac{v_{m l}^{\beta} v_{l p}^{\alpha} f_{p l}}{\hbar \bar{\omega}_{1}-\epsilon_{l p}}-\frac{f_{l m} v_{m l}^{\alpha} v_{l p}^{\beta}}{\hbar \bar{\omega}_{1}-\epsilon_{m l}}\right) \frac{v_{p n}^{\lambda}}{\hbar\left(\bar{\omega}_{2}+\bar{\omega}_{1}\right)-\epsilon_{m p}}\right. \\
& \left.+\frac{v_{n p}^{\lambda}}{\hbar\left(\bar{\omega}_{2}+\bar{\omega}_{1}\right)+\epsilon_{m p}}\left(\frac{v_{p l}^{\beta} v_{l m}^{\alpha} f_{m l}}{\hbar \bar{\omega}_{1}-\epsilon_{l m}}-\frac{f_{l p} v_{p l}^{\alpha} v_{l m}^{\beta}}{\hbar \bar{\omega}_{1}-\epsilon_{p l}}\right) \frac{v_{m n}^{\phi}}{\hbar \bar{\omega}_{s}+\epsilon_{m n}}\right] \\
& \sigma_{\phi \lambda \beta \alpha}^{\{i e e\}}\left(\omega_{3}, \omega_{2}, \omega_{1}\right)=\frac{g \sigma_{3}}{i \Omega} \frac{\hbar^{3} \gamma_{0}^{2}}{a_{0}^{2}} \sum_{l m n} \sum_{\mathbf{k}}\left\{\left(\frac{v_{n m}^{\phi}}{\hbar \omega_{s}-\epsilon_{m n}}\right)_{; \lambda} \frac{\bar{\delta}_{m l} \bar{\delta}_{l n} / \epsilon_{m l} \epsilon_{l n}}{\hbar\left(\bar{\omega}_{2}+\bar{\omega}_{1}\right)-\epsilon_{m n}}\left(\frac{v_{m l}^{\beta} v_{l n}^{\alpha} f_{n l}}{\hbar \bar{\omega}_{1}-\epsilon_{l n}}-\frac{f_{l m} v_{m l}^{\alpha} v_{l n}^{\beta}}{\hbar \bar{\omega}_{1}-\epsilon_{m l}}\right)\right. \\
& -\frac{v_{n m}^{\phi} \bar{\delta}_{m l} \bar{\delta}_{l n}}{\hbar \bar{\omega}_{s}-\epsilon_{m n}}\left[\frac{v_{m l}^{\lambda} / \epsilon_{m l}}{\hbar\left(\bar{\omega}_{2}+\bar{\omega}_{1}\right)-\epsilon_{l n}}\left(\frac{v_{l n}^{\alpha} f_{n l} / \epsilon_{l n}}{\hbar \bar{\omega}_{1}-\epsilon_{l n}}\right)_{; \beta}-\left(\frac{f_{l m} v_{m l}^{\alpha} / \epsilon_{m l}}{\hbar \bar{\omega}_{1}-\epsilon_{m l}}\right)_{; \beta} \frac{v_{l n}^{\lambda} / \epsilon_{l n}}{\hbar\left(\bar{\omega}_{2}+\bar{\omega}_{1}\right)-\epsilon_{m l}}\right] \\
& \left.-\frac{1}{\hbar \bar{\omega}_{1}} \frac{\bar{\delta}_{m l} \bar{\delta}_{l n} v_{n m}^{\phi}}{\hbar \bar{\omega}_{s}-\epsilon_{m n}}\left[\frac{v_{m l}^{\lambda} v_{l n}^{\beta} / \epsilon_{m l} \epsilon_{l n}}{\hbar\left(\bar{\omega}_{2}+\bar{\omega}_{1}\right)-\epsilon_{l n}} \frac{\partial f_{n l}}{\partial k_{\alpha}}-\frac{v_{m l}^{\beta} v_{l n}^{\lambda} / \epsilon_{m l} \epsilon_{l n}}{\hbar\left(\bar{\omega}_{2}+\bar{\omega}_{1}\right)-\epsilon_{m l}} \frac{\partial f_{l m}}{\partial k_{\alpha}}\right]\right\}, \\
& \sigma_{\phi \lambda \beta \alpha}^{\{i i e\}}\left(\omega_{3}, \omega_{2}, \omega_{1}\right)=\frac{-g \sigma_{3}}{i \Omega} \frac{\hbar^{2} \gamma_{0}^{2}}{a_{0}^{2}} \sum_{m n} \sum_{\mathbf{k}}\left[\left(\frac{v_{n m}^{\phi}}{\hbar \bar{\omega}_{s}-\epsilon_{m n}}\right)_{; \lambda} \frac{\bar{\delta}_{m n}}{\hbar\left(\bar{\omega}_{2}+\bar{\omega}_{1}\right)-\epsilon_{m n}}\left(\frac{v_{m n}^{\alpha} f_{n m} / \epsilon_{m n}}{\hbar \bar{\omega}_{1}-\epsilon_{m n}}\right)_{; \beta}\right. \\
& \left.+\left(\frac{\bar{\delta}_{m n} v_{n m}^{\phi}}{\hbar \bar{\omega}_{s}-\epsilon_{m n}}\right)_{; \lambda} \frac{v_{m n}^{\beta} /\left(\epsilon_{m n} \hbar \bar{\omega}_{1}\right)}{\hbar\left(\bar{\omega}_{2}+\bar{\omega}_{1}\right)-\epsilon_{m n}} \frac{\partial f_{n m}}{\partial k_{\alpha}}-\frac{1}{\hbar\left(\bar{\omega}_{2}+\bar{\omega}_{1}\right) \hbar \bar{\omega}_{1}} \frac{\bar{\delta}_{m n} v_{n m}^{\phi}}{\hbar \bar{\omega}_{s}-\epsilon_{m n}} \frac{v_{m n}^{\lambda}}{\epsilon_{m n}} \frac{\partial^{2} f_{n m}}{\partial k_{\beta} \partial k_{\alpha}}\right] \\
& \sigma_{\phi \lambda \beta \alpha}^{i i i}\left(\omega_{3}, \omega_{2}, \omega_{1}\right)=\frac{g \sigma_{3}}{i \Omega} \frac{\hbar \gamma_{0}^{2} / a_{0}^{2}}{\hbar \bar{\omega}_{s} \hbar\left(\bar{\omega}_{2}+\omega_{1}\right) \hbar \bar{\omega}_{1}} \sum_{n} \sum_{\mathbf{k}} \frac{\partial v_{n n}^{\phi}}{\partial k_{\lambda}} \frac{\partial^{2} f_{n}}{\partial k_{\beta} \partial k_{\alpha}},
\end{aligned}
$$

where the third-order nonlinear $2 \mathrm{D}$ conductivity unit reads $\sigma_{3} \equiv e^{4} a_{0}^{2} / 8 \gamma_{0}^{2} \hbar$, and we make use of the contracted notation $\{i e e\}=i e e+e i e+e e i,\{i i e\}=i i e+i e i+e i i$.

It is important to highlight that we made use of several permutations of dummy indices and applied integration by parts for periodic functions to derive the results shown in Eq. (A4).

As identified in Ref. 26, Eqs. (A3), and (A4) contain spurious divergences that can affect the evaluation of the nonlinear optical response of cold insulators. For example, the ee term, Eq. (A4a), exhibits spurious divergences for all $m=n$ or $m=l$. In addition to the spurious divergences, the results shown Eqs. (A2), (A3), and (A4), include the expectable Drude-like terms associated with intraband motion of electrons. As discussed in the main text, Sec. II C, the divergence appearing in the linear response, Eq. (A2), represents intraband motion of the electronic system that is responsible for the Drude peak. Beyond linear order, the problem becomes more complex and many unphysical terms can plague the calculation of the nonlinear response. Below, we introduce a straightforward, although lengthy, procedure that disentangles the physical Drude-like terms from the regular part of the nonlinear response and removes the spurious divergences.

\section{Outline of the procedure}

By making use of the effective nonlinear rank-2 tensor defined in the main text, Eq. (7), we generalize the procedure introduced in Ref. 27 to regularize the nonlinear response. We consider the case that all frequencies are taken to the DC limit simultaneously. In this limit, the analytically continued frequencies $\bar{\omega} \equiv \omega+i \eta$ can be mapped to a unique frequency $\bar{\omega}_{n}=x_{n} \varpi$, where $x_{n}$ are real numbers. This transformation allows us to consider only one limit, $\varpi \rightarrow 0$, and thus handle the divergences occurring in the DC limit. The analytic continuation of the frequencies ensures that the transformation holds, even for cases such as the nonlinear OR, where $\bar{\omega}_{2}+\bar{\omega}_{1}= \pm\left(\omega_{1}-\omega_{1}\right)+2 i \eta$. Moreover, it can be shown all divergences that arise from the combination of positive and negative frequency components are always canceled by a symmetric contribution that stems from the complementary frequency combination.

The procedure follows naturally from the definition of the effective tensor and can be summarized as follows: group tensor elements according to the number of interand intraband transitions; isolate all divergences in $\varpi$ by 
means of partial fraction decomposition with the identities

$$
\frac{1}{\hbar \varpi(\epsilon \mp \hbar \varpi)}=\frac{1}{\hbar \varpi \epsilon} \pm \frac{1}{\epsilon(\epsilon \mp \hbar \varpi)}
$$

for each divergence occurring at frequency component $\omega_{s}=\omega_{n}+\cdots+\omega_{1}$, add all terms associated with the permutations of the spatial indices, i.e. add all combinations for each value $\nu$.

\section{Isolating the divergences}

The procedure leads to a redefinition of the conductivity tensors in terms of a power series in $1 / \varpi$. The effective rank-2 tensors for the second-order process read

$$
\begin{aligned}
\bar{\sigma}_{\phi \nu}^{e e}\left(\omega_{s}\right) & \equiv \sum_{\omega_{2} \omega_{1}}^{\prime} \sum_{\lambda \alpha}^{\prime}\left(A_{\phi \lambda \alpha}^{e e}+a_{\phi \lambda \alpha}^{e e}+b_{\phi \lambda \alpha}^{e e} / \varpi\right) \\
\bar{\sigma}_{\phi \nu}^{e i}\left(\omega_{s}\right) & \equiv \sum_{\omega_{2} \omega_{1}}^{\prime} \sum_{\lambda \alpha}^{\prime}\left(A_{\phi \lambda \alpha}^{e i}+B_{\phi \lambda \alpha}^{e i} / \varpi\right) \\
\bar{\sigma}_{\phi \nu}^{i e}\left(\omega_{s}\right) & \equiv \sum_{\omega_{2} \omega_{1}}^{\prime} \sum_{\lambda \alpha}^{\prime} A_{\phi \lambda \alpha}^{i e} \\
\bar{\sigma}_{\phi \nu}^{i i}\left(\omega_{s}\right) & \equiv \sum_{\omega_{2} \omega_{1}}^{\prime} \sum_{\lambda \alpha}^{\prime} C_{\phi \lambda \alpha}^{i i} / \varpi^{2}
\end{aligned}
$$

To facilitate the identification of the regular and divergent terms, we adopt the following convention: regular terms are represented by $A$ and $a$; linear divergences by $B$ and $b$; quadratic by $C$ and $c$; cubic by $D$. In all cases, upper-case latin letters represent terms that involve the same number of interband transitions as the original tensor, whereas lower-case represent terms with one less interband transition. The elements that can be trivially shown to vanish are not displayed, e.g. $B_{\phi \lambda \beta \alpha}^{e e e}=0$. In addition, the primed summation over the frequencies is evaluated with the restriction $\omega_{s}=\omega_{n}+\ldots+\omega_{1}$, while the primed summation over spatial indices $\lambda \ldots \alpha$ respects the combinations for effective tensor index $\nu$ listed in Tab. II.

Note that the definitions of $A_{\phi \beta \alpha}^{i e}$ and $C_{\phi \beta \alpha}^{i i}$ are unchanged with respect to the original definitions in Eq. (A3), as the former contains no divergences and the latter only contains terms that diverge quadratically with the photon frequency in the DC limit. By the same token, the effective tensor for the third-order is cast as

$$
\begin{aligned}
\bar{\sigma}_{\phi \nu}^{e e e}\left(\omega_{s}\right) & \equiv \sum_{\omega_{3} \omega_{2} \omega_{1}} \sum_{\lambda \beta \alpha}^{\prime}\left(A_{\phi \lambda \beta \alpha}^{e e e}+a_{\phi \lambda \beta \alpha}^{e e e}+b_{\phi \lambda \beta \alpha}^{e e e} / \varpi\right), \\
\bar{\sigma}_{\phi \nu}^{\{i e e\}}\left(\omega_{s}\right) & \equiv \sum_{\omega_{3} \omega_{2} \omega_{1}} \sum_{\lambda \beta \alpha}^{\prime}\left(A_{\phi \lambda \beta \alpha}^{\{i e e\}}+a_{\phi \lambda \beta \alpha}^{\{i e e\}}+B_{\phi \lambda \beta \alpha}^{\{i e e\}} / \varpi\right. \\
& \left.+b_{\phi \lambda \beta \alpha}^{\{i e e\}} / \varpi+c_{\phi \lambda \beta \alpha}^{\{i e e\}} / \varpi^{2}\right), \\
\bar{\sigma}_{\phi \nu}^{\{i i e\}}\left(\omega_{s}\right) & \equiv \sum_{\omega_{3} \omega_{2} \omega_{1}}^{\prime} \sum_{\lambda \beta \alpha}^{\prime}\left(A_{\phi \lambda \beta \alpha}^{\{i i e\}}+B_{\phi \lambda \beta \alpha}^{\{i i e\}} / \varpi\right. \\
& \left.+C_{\phi \lambda \beta \alpha}^{\{i i e\}} / \varpi^{2}\right) . \\
\bar{\sigma}_{\phi \nu}^{i i i}\left(\omega_{s}\right) & \equiv \sum_{\omega_{3} \omega_{2} \omega_{1}}^{\prime} \sum_{\lambda \beta \alpha}^{\prime} D_{\phi \lambda \beta \alpha}^{i i i} / \varpi^{3},
\end{aligned}
$$

with $D_{\phi \lambda \beta \alpha}^{i i i}$ inheriting the original definition used in Eq. (A4d). We begin by identifying the explicit form of all regular terms and address the divergent terms in the following subsections, where we remove the spurious divergences and identify all nonlinear Drude-like terms. Note that throughout this process we relabeled several summation indices. The regular terms at second-order read

$$
\begin{aligned}
& A_{\phi \lambda \alpha}^{e e}=\hbar^{2} C_{2} \sum_{l m n} \sum_{\mathbf{k}} \frac{v_{n m}^{\phi} \bar{\delta}_{n m}}{\hbar\left(\bar{\omega}_{2}+\bar{\omega}_{1}\right)-\epsilon_{m n}} \frac{\bar{\delta}_{m l} \bar{\delta}_{l n}}{\epsilon_{m l} \epsilon_{l n}}\left(\frac{v_{m l}^{\lambda} v_{l n}^{\alpha} f_{n l}}{\hbar \bar{\omega}_{1}-\epsilon_{l n}}-\frac{f_{l m} v_{m l}^{\alpha} v_{l n}^{\lambda}}{\hbar \bar{\omega}_{1}-\epsilon_{m l}}\right), \\
& a_{\phi \lambda \alpha}^{e e}=-\hbar^{2} C_{2} \frac{\bar{\omega}_{1}}{\bar{\omega}_{2}+\bar{\omega}_{1}} \sum_{m n} \sum_{\mathbf{k}} \frac{v_{n n}^{\phi} \bar{\delta}_{m n} f_{m n}}{\epsilon_{m n}^{3}}\left(\frac{v_{n m}^{\lambda} v_{m n}^{\alpha}}{\hbar \bar{\omega}_{1}-\epsilon_{m n}}-\frac{v_{n m}^{\alpha} v_{m n}^{\lambda}}{\hbar \bar{\omega}_{1}+\epsilon_{m n}}\right), \\
& A_{\phi \lambda \alpha}^{e i}=-\hbar C_{2} \frac{\bar{\omega}_{2}+\bar{\omega}_{1}}{\bar{\omega}_{1}} \sum_{m n} \sum_{\mathbf{k}} \frac{v_{n m}^{\phi} v_{m n}^{\lambda}}{\hbar\left(\bar{\omega}_{2}+\bar{\omega}_{1}\right)-\epsilon_{m n}} \frac{\bar{\delta}_{m n}}{\epsilon_{m n}} \frac{\partial f_{n m}}{\partial k_{\alpha}}, \\
& A_{\phi \lambda \alpha}^{i e}=-\hbar C_{2} \sum_{m n} \sum_{\mathbf{k}} \frac{v_{n m}^{\phi} \bar{\delta}_{n m}}{\hbar\left(\bar{\omega}_{2}+\bar{\omega}_{1}\right)-\epsilon_{m n}}\left(\frac{v_{m n}^{\alpha} f_{n m} / \epsilon_{m n}}{\hbar \bar{\omega}_{1}-\epsilon_{m n}}\right)_{; \lambda},
\end{aligned}
$$

with $C_{2}=\left(g \sigma_{2} / \Omega\right)\left(\hbar \gamma_{0} / a_{0}\right)$. The regularized expressions for third-order processes read

$$
A_{\phi \lambda \beta \alpha}^{e e e}=-\hbar^{4} C_{3} \sum_{p l m n} \sum_{\mathbf{k}} \frac{\bar{\delta}_{p m} \bar{\delta}_{m l} \bar{\delta}_{l p} \bar{\delta}_{p n} \bar{\delta}_{n m}}{\epsilon_{m l} \epsilon_{l p} \epsilon_{p n}}\left[\frac{v_{n m}^{\phi}}{\hbar \bar{\omega}_{s}-\epsilon_{m n}}\left(\frac{v_{m l}^{\beta} v_{l p}^{\alpha} f_{p l}}{\hbar \bar{\omega}_{1}-\epsilon_{l p}}-\frac{f_{l m} v_{m l}^{\alpha} v_{l p}^{\beta}}{\hbar \bar{\omega}_{1}-\epsilon_{m l}}\right) \frac{v_{p n}^{\lambda}}{\hbar\left(\bar{\omega}_{2}+\bar{\omega}_{1}\right)-\epsilon_{m p}}\right.
$$




$$
\begin{aligned}
& \left.+\frac{v_{n p}^{\lambda}}{\hbar\left(\bar{\omega}_{2}+\bar{\omega}_{1}\right)+\epsilon_{m p}}\left(\frac{v_{p l}^{\beta} v_{l m}^{\alpha} f_{m l}}{\hbar \bar{\omega}_{1}-\epsilon_{l m}}-\frac{f_{l p} v_{p l}^{\alpha} v_{l m}^{\beta}}{\hbar \bar{\omega}_{1}-\epsilon_{p l}}\right) \frac{v_{m n}^{\phi}}{\hbar \bar{\omega}_{s}+\epsilon_{m n}}\right] \\
& A_{\phi \lambda \beta \alpha}^{\{i e e\}}=\hbar^{3} C_{3} \sum_{l m n} \sum_{\mathbf{k}} \bar{\delta}_{n m} \bar{\delta}_{m l} \bar{\delta}_{l n}\left\{\left(\frac{v_{n m}^{\phi}}{\hbar \bar{\omega}_{s}-\epsilon_{m n}}\right)_{; \lambda} \frac{1 / \epsilon_{m l} \epsilon_{l n}}{\hbar\left(\bar{\omega}_{2}+\bar{\omega}_{1}\right)-\epsilon_{m n}}\left(\frac{v_{m l}^{\beta} v_{l n}^{\alpha} f_{n l}}{\hbar \bar{\omega}_{1}-\epsilon_{l n}}-\frac{f_{l m} v_{m l}^{\alpha} v_{l n}^{\beta}}{\hbar \bar{\omega}_{1}-\epsilon_{m l}}\right)\right. \\
& -\frac{v_{n m}^{\phi}}{\hbar \bar{\omega}_{s}-\epsilon_{m n}}\left[\frac{v_{m l}^{\lambda} / \epsilon_{m l}}{\hbar\left(\bar{\omega}_{2}+\bar{\omega}_{1}\right)-\epsilon_{l n}}\left(\frac{v_{l n}^{\alpha} f_{n l} / \epsilon_{l n}}{\hbar \bar{\omega}_{1}-\epsilon_{l n}}\right)_{; \beta}-\left(\frac{f_{l m} v_{m l}^{\alpha} / \epsilon_{m l}}{\hbar \bar{\omega}_{1}-\epsilon_{m l}}\right)_{; \beta} \frac{v_{l n}^{\lambda} / \epsilon_{l n}}{\hbar\left(\bar{\omega}_{2}+\bar{\omega}_{1}\right)-\epsilon_{m l}}\right] \\
& -\frac{v_{n m}^{\phi}}{\epsilon_{m n} \epsilon_{m l} \epsilon_{l n}}\left[\frac{\bar{\omega}_{s} / \bar{\omega}_{1}}{\hbar \bar{\omega}_{s}-\epsilon_{m n}}\left(\frac{v_{m l}^{\lambda} v_{l n}^{\beta}}{\hbar\left(\bar{\omega}_{2}+\bar{\omega}_{1}\right)-\epsilon_{l n}} \frac{\partial f_{n l}}{\partial k_{\alpha}}-\frac{\partial f_{l m}}{\partial k_{\alpha}} \frac{v_{m l}^{\beta} v_{l n}^{\lambda}}{\hbar\left(\bar{\omega}_{2}+\bar{\omega}_{1}\right)-\epsilon_{m l}}\right)\right. \\
& \left.\left.-\frac{\bar{\omega}_{2}+\bar{\omega}_{1}}{\bar{\omega}_{1}}\left(\frac{v_{m l}^{\lambda} v_{l n}^{\beta} / \epsilon_{l n}}{\hbar\left(\bar{\omega}_{2}+\bar{\omega}_{1}\right)-\epsilon_{l n}} \frac{\partial f_{n l}}{\partial k_{\alpha}}-\frac{\partial f_{l m}}{\partial k_{\alpha}} \frac{v_{m l}^{\beta} v_{l n}^{\lambda} / \epsilon_{m l}}{\hbar\left(\bar{\omega}_{2}+\bar{\omega}_{1}\right)-\epsilon_{m l}}\right)\right]\right\} \\
& A_{\phi \lambda \beta \alpha}^{\{i i e\}}=-\hbar^{2} C_{3} \sum_{m n} \sum_{\mathbf{k}} \bar{\delta}_{m n}\left[\left(\frac{v_{n m}^{\phi}}{\hbar \bar{\omega}_{s}-\epsilon_{m n}}\right)_{; \lambda} \frac{1}{\hbar\left(\bar{\omega}_{2}+\bar{\omega}_{1}\right)-\epsilon_{m n}}\left(\frac{v_{m n}^{\alpha} f_{n m} / \epsilon_{m n}}{\hbar \bar{\omega}_{1}-\epsilon_{m n}}\right)_{; \beta}-\frac{\bar{\omega}_{s}^{2} / \bar{\omega}_{1}}{\bar{\omega}_{1}+\bar{\omega}_{2}} \frac{v_{n m}^{\phi} v_{m n}^{\lambda} / \epsilon_{m n}^{3}}{\hbar \bar{\omega}_{s}-\epsilon_{m n}} \frac{\partial^{2} f_{n m}}{\partial k_{\beta} \partial k_{\alpha}}\right. \\
& \left.+\left[\left(\frac{v_{n m}^{\phi}}{\hbar \bar{\omega}_{s}-\epsilon_{m n}}\right)_{; \lambda} \frac{\left(\bar{\omega}_{2}+\bar{\omega}_{1}\right) / \bar{\omega}_{1}}{\hbar\left(\bar{\omega}_{2}+\bar{\omega}_{1}\right)-\epsilon_{m n}}-\frac{\bar{\omega}_{s}}{\bar{\omega}_{1}}\left(\frac{v_{n m}^{\phi} / \epsilon_{m n}}{\hbar \bar{\omega}_{s}-\epsilon_{m n}}\right)_{; \lambda}\right] \frac{v_{m n}^{\beta}}{\epsilon_{m n}^{2}} \frac{\partial f_{n m}}{\partial k_{\alpha}}\right], \\
& a_{\phi \lambda \beta \alpha}^{e e e}=-\hbar^{4} C_{3} \sum_{l m n} \sum_{\mathbf{k}}\left(\frac{\bar{\delta}_{n m} \bar{\delta}_{m l} \bar{\delta}_{l n}}{\epsilon_{m n} \epsilon_{m l} \epsilon_{l n}}\{\right. \\
& \frac{\bar{\omega}_{2}+\bar{\omega}_{1}}{\bar{\omega}_{s}}\left[\left(\frac{v_{n l}^{\beta} v_{l m}^{\alpha} f_{l m}}{\hbar \bar{\omega}_{1}+\epsilon_{m l}}-\frac{f_{n l} v_{n l}^{\alpha} v_{l m}^{\beta}}{\hbar \bar{\omega}_{1}+\epsilon_{l n}}\right) \frac{v_{m n}^{\lambda} v_{n n}^{\phi} / \epsilon_{m n}}{\hbar \bar{\omega}_{s}+\epsilon_{m n}}+\frac{v_{n n}^{\phi} v_{n m}^{\lambda} / \epsilon_{m n}}{\hbar \bar{\omega}_{s}-\epsilon_{m n}}\left(\frac{v_{n l}^{\beta} v_{l m}^{\alpha} f_{n l}}{\hbar \bar{\omega}_{1}-\epsilon_{l n}}-\frac{f_{l m} v_{n l}^{\alpha} v_{l m}^{\beta}}{\hbar \bar{\omega}_{1}+\epsilon_{m l}}\right)\right] \\
& \left.+\frac{\bar{\omega}_{1}}{\bar{\omega}_{s}} \frac{v_{n n}^{\phi}}{\epsilon_{m n}}\left[\left(\frac{v_{n l}^{\beta} v_{l m}^{\alpha} f_{l m} / \epsilon_{m l}}{\hbar \bar{\omega}_{1}+\epsilon_{m l}}-\frac{f_{n l} v_{n l}^{\alpha} v_{l m}^{\beta} / \epsilon_{l n}}{\hbar \bar{\omega}_{1}+\epsilon_{l n}}\right) v_{m n}^{\lambda}-v_{n m}^{\lambda}\left(\frac{v_{m l}^{\beta} v_{l n}^{\alpha} f_{n l} / \epsilon_{l n}}{\hbar \bar{\omega}_{1}-\epsilon_{l n}}-\frac{f_{l m} v_{m l}^{\alpha} v_{l n}^{\beta} / \epsilon_{m l}}{\hbar \bar{\omega}_{1}-\epsilon_{m l}}\right)\right]\right\} \\
& -\frac{\bar{\delta}_{m l} \bar{\delta}_{m n}}{\epsilon_{m l}^{2} \epsilon_{m n}}\left[\frac{\bar{\omega}_{1}}{\bar{\omega}_{2}+\bar{\omega}_{1}}\left(\frac{v_{n m}^{\phi} v_{m n}^{\lambda}}{\hbar \bar{\omega}_{s}-\epsilon_{m n}}+\frac{v_{n m}^{\lambda} v_{m n}^{\phi}}{\hbar \bar{\omega}_{s}+\epsilon_{m n}}\right) \frac{f_{l m}}{\epsilon_{m l}}\left(\frac{v_{m l}^{\beta} v_{l m}^{\alpha}}{\hbar \bar{\omega}_{1}+\epsilon_{m l}}-\frac{v_{m l}^{\alpha} v_{l m}^{\beta}}{\hbar \bar{\omega}_{1}-\epsilon_{m l}}\right)\right. \\
& \left.\left.-\frac{\bar{\omega}_{2}+\bar{\omega}_{1}}{\bar{\omega}_{s}}\left(\frac{v_{n m}^{\phi} v_{m n}^{\lambda}}{\hbar \bar{\omega}_{s}-\epsilon_{m n}}+\frac{v_{n m}^{\lambda} v_{m n}^{\phi}}{\hbar \bar{\omega}_{s}+\epsilon_{m n}}\right) \frac{f_{l m}}{\epsilon_{m n}} \frac{v_{m l}^{\beta} v_{l m}^{\alpha}-v_{m l}^{\alpha} v_{l m}^{\beta}}{\epsilon_{m l}}\right]\right) \text {, } \\
& a_{\phi \lambda \beta \alpha}^{\{i e e\}}=\hbar^{3} C_{3} \sum_{m n} \sum_{\mathbf{k}} \bar{\delta}_{m n}\left(-\frac{\bar{\omega}_{1}^{2}\left(v_{n n}^{\phi}\right)_{; \lambda}}{\bar{\omega}_{s}\left(\bar{\omega}_{2}+\bar{\omega}_{1}\right)} \frac{f_{n m}}{\epsilon_{m n}^{4}}\left(\frac{v_{n m}^{\beta} v_{m n}^{\alpha}}{\hbar \bar{\omega}_{1}-\epsilon_{m n}}-\frac{v_{n m}^{\alpha} v_{m n}^{\beta}}{\hbar \bar{\omega}_{1}+\epsilon_{m n}}\right)\right. \\
& +\frac{\bar{\omega}_{1}}{\bar{\omega}_{s}} \frac{v_{n n}^{\phi}}{\epsilon_{m n}}\left[\frac{v_{n m}^{\lambda}}{\hbar\left(\bar{\omega}_{1}+\bar{\omega}_{1}\right)-\epsilon_{m n}}\left(\frac{v_{m n}^{\alpha} f_{n m} / \epsilon_{m n}^{2}}{\hbar \bar{\omega}_{1}-\epsilon_{m n}}\right)_{; \beta}-\left(\frac{v_{n m}^{\alpha} f_{n m} / \epsilon_{m n}^{2}}{\hbar \bar{\omega}_{1}+\epsilon_{m n}}\right)_{; \beta} \frac{v_{m n}^{\lambda}}{\hbar\left(\bar{\omega}_{1}+\bar{\omega}_{1}\right)+\epsilon_{m n}}\right] \\
& -\frac{\left(\bar{\omega}_{2}+\bar{\omega}_{1}\right)}{\bar{\omega}_{s}} \frac{v_{n n}^{\phi}}{\epsilon_{m n}^{2}}\left[\frac{v_{n m}^{\lambda}}{\hbar\left(\bar{\omega}_{2}+\bar{\omega}_{1}\right)-\epsilon_{m n}}\left(\frac{v_{m n}^{\alpha} f_{n m}}{\epsilon_{m n}^{2}}\right)_{; \beta}+\left(\frac{v_{n m}^{\alpha} f_{n m}}{\epsilon_{m n}^{2}}\right)_{; \beta} \frac{v_{m n}^{\lambda}}{\hbar\left(\bar{\omega}_{2}+\bar{\omega}_{1}\right)-\epsilon_{m n}}\right] \\
& \left.+\frac{\left(\bar{\omega}_{2}+\bar{\omega}_{1}\right)^{2}}{\bar{\omega}_{s} \bar{\omega}_{1}} \frac{v_{n n}^{\phi}}{\epsilon_{m n}^{4}} \frac{\partial f_{n m}}{\partial k_{\alpha}}\left(\frac{v_{n m}^{\lambda} v_{m n}^{\beta}}{\hbar\left(\bar{\omega}_{2}+\bar{\omega}_{1}\right)-\epsilon_{m n}}+\frac{v_{n m}^{\beta} v_{m n}^{\lambda}}{\hbar\left(\bar{\omega}_{2}+\bar{\omega}_{1}\right)+\epsilon_{m n}}\right)\right) \text {. }
\end{aligned}
$$

where $C_{3}=\left(g \sigma_{3} / i \Omega\right)\left(\gamma_{0}^{2} / a_{0}^{2}\right)$. These expressions are regular at all frequencies and include all terms necessary to characterize the nonlinear response of cold insulators up to third-order.

\section{Divergences: spurious and Drude-like}

Having identified all divergent terms, we explicitly remove the spurious terms by showing that these terms vanish in the calculation of the effective tensor and also identify the nonlinear contributions to the Drude-like terms. We start by addressing the second-order response and then proceed to the third-order. 


\section{a. Quadratic response}

At second-order, the current density response contains divergences in three terms, namely in the $e e$, ei, and $i i$ terms. The divergences in the purely interband term, $b_{\phi \lambda \alpha}^{e e}$, are immediately shown to vanish for all cases with $\lambda=\alpha$, i.e. $\nu=\{1,2,3\}$. For the remaining cases, $\nu=\{4,5,6\}$, it is sufficient to consider the following combination

$$
\frac{b_{\phi \lambda \alpha}^{e e}}{\varpi}+\frac{b_{\phi \alpha \lambda}^{e e}}{\varpi}=\frac{\hbar^{2} C_{2}}{\hbar\left(\bar{\omega}_{2}+\bar{\omega}_{1}\right)} \sum_{m n} \sum_{\mathbf{k}} \frac{v_{n n}^{\phi} \bar{\delta}_{m n} f_{m n}}{\epsilon_{m n}} \frac{v_{n m}^{\lambda} v_{m n}^{\alpha}-v_{n m}^{\alpha} v_{m n}^{\lambda}-v_{n m}^{\alpha} v_{m n}^{\lambda}+v_{n m}^{\lambda} v_{m n}^{\alpha}}{\epsilon_{m n}^{2}}=0
$$

thus showing that all divergences in the purely interband term are vanish. Upon decomposition of the regular and divergent parts of the $e i$ term, we verify that

$$
\frac{B_{\phi \lambda \alpha}^{e i}}{\varpi}=\frac{\hbar C_{2}}{\hbar \bar{\omega}_{1}} \sum_{m n} \sum_{\mathbf{k}} \frac{\bar{\delta}_{m n} v_{n m}^{\phi} v_{m n}^{\lambda}}{\epsilon_{m n}^{2}} \frac{\partial f_{n m}}{\partial k_{\alpha}}
$$

is a natural second-order Drude-like term that contributes only to the response of metallic systems or doped insulators. The purely intraband term $i i$ does not contain any regular parts and defines the quadratic Drude-like peak

$$
\frac{C_{\phi \lambda \alpha}^{i i}}{\varpi^{2}}=\frac{C_{2}}{\hbar\left(\bar{\omega}_{2}+\bar{\omega}_{1}\right) \hbar \bar{\omega}_{1}} \sum_{n} \sum_{\mathbf{k}} v_{n n}^{\phi} \frac{\partial^{2} f_{n}}{\partial k_{\lambda} \partial k_{\alpha}}
$$

\section{b. 3rd-order purely interband and purely intraband}

At third-order, the separation of the natural contributions to the nonlinear Drude peak from the spurious divergences is not trivial, particularly in processes involving inter- and intraband transitions. We start by addressing the spurious divergences in the purely interband contribution

$$
\begin{aligned}
\frac{b_{\phi \nu}^{e e e}}{\varpi} & =-\hbar^{4} C_{3} \sum_{l m n} \sum_{\lambda \beta \alpha}^{\prime} \sum_{\mathbf{k}}\left\{\frac{\bar{\delta}_{n m} \bar{\delta}_{m l} \bar{\delta}_{l n}}{\epsilon_{m n}^{2} \epsilon_{m l}^{2} \epsilon_{l n}^{2}} \frac{v_{n n}^{\phi}}{\hbar \bar{\omega}_{s}}\left[f_{l m} \epsilon_{l n}\left(V_{n l m}^{\beta \alpha \lambda}+V_{n m l}^{\lambda \alpha \beta}\right)-f_{n l} \epsilon_{m l}\left(V_{n l m}^{\alpha \beta \lambda}+V_{n m l}^{\lambda \alpha \beta}\right)\right]\right. \\
& \left.+\frac{\bar{\delta}_{m l} \bar{\delta}_{l n}}{\epsilon_{m l}^{2} \epsilon_{m n}^{2}} f_{l m} \frac{v_{n m}^{\phi} v_{m n}^{\lambda}-v_{n m}^{\lambda} v_{m n}^{\phi}}{\hbar \bar{\omega}_{1}} \frac{v_{m l}^{\beta} v_{l m}^{\alpha}-v_{m l}^{\alpha} v_{l m}^{\beta}}{\epsilon_{m l}}\right\},
\end{aligned}
$$

where $V_{n m l}^{\lambda \alpha \beta} \equiv v_{n m}^{\lambda} v_{m l}^{\beta} v_{l n}^{\alpha}$. The latter arises from the cases where $m=l$ in Eq. (A4a) (note that we replace $p \rightarrow m$ to recover the $l$ index) and vanishes for all $\{l m n\}$ by considering all combinations to the effective tensor. To prove that the former [associated with $m=n$ terms in Eq. (A4a)] vanishes, we begin by expanding the Fermi energy differences, add a second copy with interchanged indices $m, l$ and then make use of the combinations that define the effective tensor

$$
\begin{aligned}
\frac{b_{\phi \nu}^{e e e}}{\varpi} & =-\frac{\hbar^{4} C_{3}}{2} \sum_{l m n} \sum_{\mathbf{k}} \frac{\bar{\delta}_{n m} \bar{\delta}_{m l} \bar{\delta}_{l n}}{\epsilon_{m n}^{2} \epsilon_{m l}^{2} \epsilon_{l n}^{2}} \frac{v_{n n}^{\phi}}{\hbar \bar{\omega}_{s}} \sum_{\lambda \beta \alpha}^{\prime}\left[f_{l} \epsilon_{m n}\left(V_{n l m}^{\beta \alpha \lambda}+V_{n m l}^{\lambda \alpha \beta}-V_{n m l}^{\beta \alpha \lambda}-V_{n l m}^{\lambda \alpha \beta}\right)\right. \\
& \left.+\left(f_{m} \epsilon_{l n}-f_{n} \epsilon_{m n}\right)\left(V_{n l m}^{\beta \alpha \lambda}+V_{n m l}^{\lambda \alpha \beta}-V_{n m l}^{\beta \alpha \lambda}-V_{n l m}^{\lambda \alpha \beta}\right)\right]=0,
\end{aligned}
$$

that vanishes for all effective tensors. There are no quadratic divergences and the purely intraband contribution has no spurious divergences, it is physical and defines the cubic Drude-like term for third-order response

$$
\begin{gathered}
\frac{D_{\phi \lambda \beta \alpha}^{i i i}}{\varpi^{3}}=\frac{\hbar C_{3}}{\hbar \bar{\omega}_{s} \hbar\left(\bar{\omega}_{2}+\bar{\omega}_{1}\right) \hbar \bar{\omega}_{1}} \sum_{n} \sum_{\mathbf{k}} \frac{\partial v_{n n}^{\phi}}{\partial k_{\lambda}} \frac{\partial^{2} f_{n}}{\partial k_{\beta} \partial k_{\alpha}} . \\
\text { c. } \quad \text { 3rd-order mixed }\{\text { iee }\}-\text { two inter- and one intraband }
\end{gathered}
$$

The analysis of the divergent terms involving three bands can be facilitated, provided that we consider not the individual tensor, but rather $B_{\phi \lambda \beta \alpha}^{\{i e e\}} \rightarrow\left(B_{\phi \lambda \beta \alpha}^{\{i e e\}}+B_{\phi \beta \lambda \alpha}^{\{i e e\}}\right) / 2$. The permutation of indices $\{\lambda, \beta\}$ is consistent with 
all effective tensor elements. Using this transformation, the divergence involving three band processes defines a contribution for a linear Drude-like term in the third-order response.

$$
\frac{B_{\phi \lambda \beta \alpha}^{\{i e e\}}+B_{\phi \beta \lambda \alpha}^{\{i e e\}}}{2 \varpi}=-\frac{\hbar^{3} C_{3}}{2 \hbar \bar{\omega}_{1}} \sum_{l m n} \sum_{\mathbf{k}} \frac{v_{n m}^{\phi} \bar{\delta}_{n m} \bar{\delta}_{m l} \bar{\delta}_{l n}}{\epsilon_{m n} \epsilon_{m l} \epsilon_{l n}} \frac{v_{m l}^{\lambda} v_{l n}^{\beta}+v_{m l}^{\beta} v_{l n}^{\lambda}}{\epsilon_{m l} \epsilon_{l n}}\left(\frac{\partial f_{n}}{\partial k_{\alpha}} \epsilon_{m l}+\frac{\partial f_{m}}{\partial k_{\alpha}} \epsilon_{l n}-\frac{\partial f_{l}}{\partial k_{\alpha}} \epsilon_{m n}\right) .
$$

The origin of this can be traced to the eei branch of the density matrix, that is usually discarded from the onset in the calculation of the response of cold insulators. ${ }^{26}$

Proceeding to the divergence present in the processes involving only two bands, we find

$$
\begin{aligned}
\frac{b_{\phi \lambda \beta \alpha}^{\{i e e\}}}{\varpi} & =\hbar^{3} C_{3} \sum_{m n} \sum_{\mathbf{k}} \frac{\bar{\delta}_{m n}}{\hbar \bar{\omega}_{s}}\left\{\frac{1}{x_{2}} \frac{\partial v_{n n}^{\phi}}{\partial k_{\lambda}} \frac{v_{n m}^{\beta} v_{m n}^{\alpha}+v_{n m}^{\alpha} v_{m n}^{\beta}}{\epsilon_{m n}^{4}} f_{n m}+\frac{v_{n n}^{\phi}}{\epsilon_{m n}^{2}}\left[v_{n m}^{\lambda}\left(\frac{v_{m n}^{\alpha} f_{n m}}{\epsilon_{m n}^{2}}\right)_{; \beta}+\left(\frac{v_{n m}^{\alpha} f_{n m}}{\epsilon_{m n}^{2}}\right)_{; \beta} v_{m n}^{\lambda}\right]\right. \\
& \left.+x_{2} \frac{v_{n n}^{\phi}}{\epsilon_{m n}^{2}} \frac{v_{n m}^{\lambda} v_{m n}^{\beta}+v_{n m}^{\beta} v_{m n}^{\lambda}}{\epsilon_{m n}^{2}} \frac{\partial f_{n m}}{\partial \beta}\right\} .
\end{aligned}
$$

The explicit dependence of the response on $1 / x_{2} \equiv \bar{\omega}_{1} /\left(\bar{\omega}_{2}+\bar{\omega}_{1}\right)$ and $x_{2} \equiv\left(\bar{\omega}_{2}+\bar{\omega}_{1}\right) / \bar{\omega}_{1}$ indicates that we must consider with care the permutations of frequencies whenever $\omega_{2} \neq \omega_{1}$

$$
\begin{aligned}
\frac{b_{\phi \lambda \beta \alpha}^{\{i e e\}}}{\varpi} & \equiv \frac{b_{\phi \lambda \beta \alpha}^{\{i e e\}}\left(\omega_{3}, \omega_{2}, \omega_{1}\right)+b_{\phi \lambda \beta \alpha}^{\{i e e\}}\left(\omega_{3}, \omega_{1}, \omega_{2}\right)}{2 \varpi} \\
& =\frac{\hbar^{3} C_{3}}{2} \sum_{m n} \sum_{\mathbf{k}} \frac{\bar{\delta}_{m n}}{\hbar \bar{\omega}_{s}}\left\{\frac{\partial v_{n n}^{\phi}}{\partial k_{\lambda}} \frac{v_{n m}^{\beta} v_{m n}^{\alpha}+v_{n m}^{\alpha} v_{m n}^{\beta}}{\epsilon_{m n}^{4}} f_{n m}+2 \frac{v_{n n}^{\phi}}{\epsilon_{m n}^{2}}\left[v_{n m}^{\lambda}\left(\frac{v_{m n}^{\alpha} f_{n m}}{\epsilon_{m n}^{2}}\right)_{; \beta}+\left(\frac{v_{n m}^{\alpha} f_{n m}}{\epsilon_{m n}^{2}}\right)_{; \beta} v_{m n}^{\lambda}\right]\right. \\
& \left.+\frac{\left(\bar{\omega}_{1}+\bar{\omega}_{2}\right)^{2}}{\bar{\omega}_{1} \bar{\omega}_{2}} \frac{v_{n n}^{\phi}}{\epsilon_{m n}^{2}} \frac{v_{n m}^{\lambda} v_{m n}^{\beta}+v_{n m}^{\beta} v_{m n}^{\lambda}}{\epsilon_{m n}^{2}} \frac{\partial f_{n m}}{\partial \beta}\right\} .
\end{aligned}
$$

As in the three-band process, this term represents a physical contribution to the response of metallic systems and doped semiconductors that results in an additional linear Drude-like term for the cubic response function.

The remaining term in this class of processes

$$
\frac{c_{\phi \lambda \beta \alpha}^{\{i e e\}}}{\varpi^{2}}=-\frac{\hbar^{3} C_{3}}{\hbar \bar{\omega}_{s} \hbar \bar{\omega}_{1}} \sum_{m n} \sum_{\mathbf{k}} v_{n n}^{\phi} \bar{\delta}_{m n}\left[\left(f_{n m} \frac{v_{n m}^{\beta} v_{m n}^{\alpha}-v_{n m}^{\alpha} v_{m n}^{\beta}}{\epsilon_{m n}^{3}}\right)_{; \lambda}+\frac{\partial f_{n m}}{\partial k_{\alpha}} \frac{v_{n m}^{\lambda} v_{m n}^{\beta}-v_{n m}^{\beta} v_{m n}^{\lambda}}{\epsilon_{m n}^{3}}\right]=0
$$

is shown to vanish for all effective tensor, as the numerators in the form $v_{n m}^{\beta} v_{m n}^{\alpha}-v_{n m}^{\alpha} v_{m n}^{\beta}$ cancel for all $\nu$.

\section{d. 3rd-order mixed $\{$ iie $\}$ - one inter- and two intraband}

The last divergences involve a single interband transition and two intraband processes. Such terms are never considered in Ref. 26, as these divergences stem from the iei and eii branches of the density matrix, that are discarded from the onset in the response of cold insulators. The linear divergences represent a physical contribution to the Drude-peak

$$
\frac{B_{\phi \nu}^{\{i i e\}}}{\varpi}=-\frac{\hbar^{2} C_{3}}{\hbar \bar{\omega}_{1}} \sum_{m n} \sum_{\mathbf{k}} \sum_{\lambda \beta \alpha}^{\prime} \bar{\delta}_{m n}\left[\left(\frac{v_{n m}^{\phi}}{\epsilon_{m n}}\right)_{; \lambda} \frac{v_{m n}^{\beta}}{\epsilon_{m n}^{2}} \frac{\partial f_{n m}}{\partial k_{\alpha}}+\frac{\bar{\omega}_{s}}{\bar{\omega}_{2}+\bar{\omega}_{1}} \frac{v_{n m}^{\phi} v_{n m}^{\lambda}}{\epsilon_{m n}^{3}} \frac{\partial^{2} f_{n m}}{\partial k_{\beta} \partial k_{\alpha}}\right] .
$$

In contrast, the quadratic divergence is spurious and shown to vanish, upon integration, for all effective tensors upon summation of pairs of bands

$$
\begin{aligned}
\frac{C_{\phi \nu}^{\{i i e\}}}{\varpi^{2}} & =-\frac{\hbar^{2} C_{3}}{\hbar\left(\bar{\omega}_{2}+\bar{\omega}_{1}\right) \hbar \bar{\omega}_{1}} \sum_{m n} \sum_{\mathbf{k}} \sum_{\lambda \beta \alpha}^{\prime} \bar{\delta}_{m n} \frac{v_{n m}^{\phi} v_{m n}^{\lambda}}{\epsilon_{m n}^{2}} \frac{\partial^{2} f_{n m}}{\partial k_{\beta} \partial k_{\alpha}} \\
& =-\frac{\hbar^{2} C_{3} / 2}{\hbar\left(\bar{\omega}_{2}+\bar{\omega}_{1}\right) \hbar \bar{\omega}_{1}} \sum_{m n} \sum_{\mathbf{k}} \sum_{\lambda \beta \alpha}^{\prime} \bar{\delta}_{m n} \frac{v_{n m}^{\phi} v_{m n}^{\lambda}-v_{m n}^{\phi} v_{n m}^{\lambda}}{\epsilon_{m n}^{2}} \frac{\partial^{2} f_{n m}}{\partial k_{\beta} \partial k_{\alpha}}=0 .
\end{aligned}
$$


These results isolate and identify all nonlinear contributions to the Drude peak and by removing all spurious divergences show that only odd powers of the frequency can contribute to the nonlinear Drude-like response.

*fh@nano.aau.dk

† tgp@nano.aau.dk

1 Y. R. Shen, The Principles of Nonlinear Optics (WileyInterscience, 2002).

2 R. W. Boyd, Nonlinear Optics, 3rd ed. (Elsevier Science Publishing Co Inc, 2008).

3 T. Gu, N. Petrone, J. F. McMillan, A. van der Zande, M. Yu, G. Q. Lo, D. L. Kwong, J. Hone, and C. W. Wong, Nat. Photon. 6, 554 (2012).

${ }^{4}$ E. Hendry, P. J. Hale, J. Moger, A. K. Savchenko, and S. A. Mikhailov, Phys. Rev. Lett. 105, 097401 (2010).

5 S. A. Mikhailov, Europhysics Letters (EPL) 79, 27002 (2007).

${ }^{6}$ K. L. Ishikawa, Phys. Rev. B 82, 201402 (2010).

7 H. K. Avetissian, A. K. Avetissian, G. F. Mkrtchian, and K. V. Sedrakian, Phys. Rev. B 85, 115443 (2012).

8 S. Wu, S. Buckley, J. R. Schaibley, L. Feng, J. Yan, D. G. Mandrus, F. Hatami, W. Yao, J. Vučković, A. Majumdar, and X. Xu, Nat. 520, 69 (2015).

9 L. A. Chizhova, F. Libisch, and J. Burgdörfer, Phys. Rev. B 94, 075412 (2016).

10 S. A. Mikhailov and K. Ziegler, J. Phys. Condens. Matter 20, 384204 (2008).

11 S.-Y. Hong, J. I. Dadap, N. Petrone, P.-C. Yeh, J. Hone, and R. M. Osgood, Phys. Rev. X 3, 021014 (2013).

12 N. Kumar, J. Kumar, C. Gerstenkorn, R. Wang, H.-Y. Chiu, A. L. Smirl, and H. Zhao, Phys. Rev. B 87, 121406 (2013).

13 Y. Li, Y. Rao, K. F. Mak, Y. You, S. Wang, C. R. Dean, and T. F. Heinz, Nano Lett. 13, 3329 (2013).

${ }^{14}$ H. Zeng, G.-B. Liu, J. Dai, Y. Yan, B. Zhu, R. He, L. Xie, S. Xu, X. Chen, W. Yao, and X. Cui, Sci. Rep. 3, 1608 (2013).

15 H. K. Avetissian, G. F. Mkrtchian, K. G. Batrakov, S. A. Maksimenko, and A. Hoffmann, Phys. Rev. B 88, 165411 (2013).

16 C. Janisch, Y. Wang, D. Ma, N. Mehta, A. L. Elías, N. Perea-López, M. Terrones, V. Crespi, and Z. Liu, Sci. Rep. 4, 5530 (2015).

17 G. Wang, X. Marie, I. Gerber, T. Amand, D. Lagarde, L. Bouet, M. Vidal, A. Balocchi, and B. Urbaszek, Phys. Rev. Lett. 114, 097403 (2015).

18 X. Yin, Z. Ye, D. A. Chenet, Y. Ye, K. O'Brien, J. C. Hone, and X. Zhang, Sci. 344, 488 (2014).

19 D. J. Clark, V. Senthilkumar, C. T. Le, D. L. Weerawarne, B. Shim, J. I. Jang, J. H. Shim, J. Cho, Y. Sim, M.-J. Seong, S. H. Rhim, A. J. Freeman, K.-H. Chung, and Y. S. Kim, Phys. Rev. B 92, 121409 (2015).

20 I. Al-Naib, J. E. Sipe, and M. M. Dignam, New J. Phys. 17, 113018 (2015).

21 L. A. Chizhova, F. Libisch, and J. Burgdörfer, Phys. Rev. B 95, 085436 (2017).

${ }^{22}$ F. Hipolito and V. M. Pereira, 2D Mater. 4, 021027 (2017).

23 J. W. McIver, D. Hsieh, H. Steinberg, P. Jarillo-Herrero, and N. Gedik, Nat. Nanotechnol. 7, 96 (2011).

24 A. Taghizadeh, F. Hipolito, and T. G. Pedersen, Phys. Rev. B 96, 195413 (2017).

25 A. Taghizadeh and T. G. Pedersen, Phys. Rev. B 97, 205432 (2018).

26 C. Aversa and J. E. Sipe, Phys. Rev. B 52, 14636 (1995).

27 F. Hipolito and T. G. Pedersen, Phys. Rev. B 97, 035431 (2018).

28 J. L. Cheng, N. Vermeulen, and J. E. Sipe, Opt. Express 22, 15868 (2014).

29 S. A. Mikhailov, Phys. Rev. B 90, 241301 (2014).

30 G. Soavi, G. Wang, H. Rostami, D. G. Purdie, D. De Fazio, T. Ma, B. Luo, J. Wang, A. K. Ott, D. Yoon, S. A. Bourelle, J. E. Muench, I. Goykhman, S. Dal Conte, M. Celebrano, A. Tomadin, M. Polini, G. Cerullo, and A. C. Ferrari, Nat. Nanotechnol. 13, 583 (2018).

31 T. G. Pedersen and K. Pedersen, Phys. Rev. B 79, 035422 (2009).

32 S. Y. Zhou, G.-H. Gweon, a. V. Fedorov, P. N. First, W. A. de Heer, D.-H. Lee, F. Guinea, A. H. Castro Neto, and A. Lanzara, Nat. Mater. 6, 916 (2007).

33 C. Riedl, C. Coletti, T. Iwasaki, A. A. Zakharov, and U. Starke, Phys. Rev. Lett. 103, 246804 (2009).

34 C. R. Woods, L. Britnell, A. Eckmann, R. S. Ma, J. C. Lu, H. M. Guo, X. Lin, G. L. Yu, Y. Cao, R. V. Gorbachev, A. V. Kretinin, J. Park, L. A. Ponomarenko, M. I. Katsnelson, Y. N. Gornostyrev, K. Watanabe, T. Taniguchi, C. Casiraghi, H.-j. J. Gao, A. K. Geim, and K. S. Novoselov, Nat. Phys. 10, 451 (2014).

35 S. J. Brun and T. G. Pedersen, Phys. Rev. B 91, 205405 (2015).

36 D. C. Elias, R. R. Nair, T. M. G. Mohiuddin, S. V. Morozov, P. Blake, M. P. Halsall, A. C. Ferrari, D. W. Boukhvalov, M. I. Katsnelson, A. K. Geim, and K. S. Novoselov, Sci. 323, 610 (2009).

37 R. Balog, B. Jørgensen, L. Nilsson, M. Andersen, E. Rienks, M. Bianchi, M. Fanetti, E. Lægsgaard, A. Baraldi, S. Lizzit, Z. Sljivancanin, F. Besenbacher, B. Hammer, T. G. Pedersen, P. Hofmann, and L. Hornekær, Nat. Mater. 9, 315 (2010).

38 J. Son, S. Lee, S. J. Kim, B. C. Park, H.-k. Lee, S. J. Kim, and J. H. Kim, Nat. Commun. 7, 13261 (2016).

39 A. L. Fetter and J. D. Walecka, Quantum Theory of ManyParticle Systems, dover ed. (Dover Publication, Inc., Mineola, New York, 1971).

${ }^{40}$ L. Wirtz, A. Marini, and A. Rubio, Phys. Rev. Lett. 96, 126104 (2006).

41 M. Grüning and C. Attaccalite, Phys. Rev. B 89, 081102 (2014).

42 T. G. Pedersen, Phys. Rev. B 92, 235432 (2015).

43 G. A. Wagoner, P. D. Persans, E. A. Van Wagenen, and G. M. Korenowski, J. Opt. Soc. Am. B 15, 1017 (1998).

44 A. Splendiani, L. Sun, Y. Zhang, T. Li, J. Kim, C.-Y. Chim, G. Galli, and F. Wang, Nano Lett. 10, 1271 (2010).

${ }^{45}$ H. Zeng, J. Dai, W. Yao, D. Xiao, and X. Cui, Nat. 
Nanotechnol. 7, 490 (2012).

46 T. Jiang, H. Liu, D. Huang, S. Zhang, Y. Li, X. Gong, Y.-R. Shen, W.-T. Liu, and S. Wu, Nat. Nanotechnol. 9, 825 (2014).

47 X. Wang, A. M. Jones, K. L. Seyler, V. Tran, Y. Jia, H. Zhao, H. Wang, L. Yang, X. Xu, and F. Xia, Nat. Nanotechnol. 10, 517 (2015).

48 M. L. Trolle, Y.-C. Tsao, K. Pedersen, and T. G. Pedersen, Phys. Rev. B 92, 161409 (2015).

${ }^{49}$ L. Li, J. Kim, C. Jin, G. J. Ye, D. Y. Qiu, F. H. da Jornada, Z. Shi, L. Chen, Z. Zhang, F. Yang, K. Watanabe, T. Taniguchi, W. Ren, S. G. Louie, X. H. Chen, Y. Zhang, and F. Wang, Nat. Nanotechnol. 12, 21 (2016).

50 G. Zhang, S. Huang, A. Chaves, C. Song, V. O. Özçelik, T. Low, and H. Yan, Nat. Commun. 8, 14071 (2017).

51 D. C. Elias, R. V. Gorbachev, A. S. Mayorov, S. V. Morozov, A. A. Zhukov, P. Blake, L. A. Ponomarenko, I. V. Grigorieva, K. S. Novoselov, F. Guinea, and A. K. Geim, Nat. Phys. 7, 701 (2011).

52 C.-H. Park, F. Giustino, C. D. Spataru, M. L. Cohen, and S. G. Louie, Nano Lett. 9, 4234 (2009).

53 C. Hwang, C.-H. Park, D. A. Siegel, A. V. Fedorov, S. G. Louie, and A. Lanzara, Phys. Rev. B 84, 125422 (2011).

54 A. G. Grushin, B. Valenzuela, and M. A. H. Vozmediano, Phys. Rev. B 80, 155417 (2009).

55 N. M. R. Peres, R. M. Ribeiro, and A. H. Castro Neto, Phys. Rev. Lett. 105, 055501 (2010).

56 T. Stroucken, J. H. Grönqvist, and S. W. Koch, Phys. Rev. B 84, 205445 (2011).

57 M. Breusing, S. Kuehn, T. Winzer, E. Malić, F. Milde, N. Severin, J. P. Rabe, C. Ropers, A. Knorr, and T. Elsaesser, Phys. Rev. B 83, 153410 (2011).

58 D. Sun, G. Aivazian, A. M. Jones, J. S. Ross, W. Yao, D. Cobden, and X. Xu, Nat. Nanotechnol. 7, 114 (2012).

59 E. Malic, T. Winzer, E. Bobkin, and A. Knorr, Phys. Rev. B 84, 205406 (2011).

${ }^{60}$ N. Sule, K. J. Willis, S. C. Hagness, and I. Knezevic, Phys. Rev. B 90, 045431 (2014).

61 F. T. Vasko, V. V. Mitin, V. Ryzhii, and T. Otsuji, Phys. Rev. B 86, 235424 (2012).

62 H. K. Avetissian and G. F. Mkrtchian, Phys. Rev. B 97, 115454 (2018).

63 H. K. Avetissian, A. K. Avetissian, B. R. Avchyan, and G. F. Mkrtchian, J. Physics: Condensed Matter 30, 185302 (2018).

${ }^{64}$ G. L. Yu, R. Jalil, B. Belle, A. S. Mayorov, P. Blake, F. Schedin, S. V. Morozov, L. A. Ponomarenko, F. Chiappini, S. Wiedmann, U. Zeitler, M. I. Katsnelson, A. K. Geim, K. S. Novoselov, and D. C. Elias, PNAS 110, 3282 (2013).

65 T. Stauber, P. Parida, M. Trushin, M. V. Ulybyshev, D. L. Boyda, and J. Schliemann, Phys. Rev. Lett. 118, 266801 (2017).

66 D. Sun, C. Divin, M. Mihnev, T. Winzer, E. Malic, A. Knorr, J. E. Sipe, C. Berger, W. A. de Heer, P. N. First, and T. B. Norris, New J. Phys. 14, 105012 (2012).

67 N. M. R. Peres, Rev. Mod. Phys. 82, 2673 (2010).

68 C. H. Park and S. G. Louie, Nano Lett. 10, 426 (2010).

${ }^{69}$ L. Ju, L. Wang, T. Cao, T. Taniguchi, K. Watanabe, S. G. Louie, F. Rana, J. Park, J. Hone, F. Wang, and P. L. McEuen, Sci. 358, 907 (2017).

70 J. Horng, C.-F. Chen, B. Geng, C. Girit, Y. Zhang, Z. Hao, H. A. Bechtel, M. C. Martin, A. Zettl, M. F.
Crommie, Y. R. Shen, and F. Wang, Phys. Rev. B 83 165113 (2011).

71 T. Stauber, N. M. R. Peres, and A. K. Geim, Phys. Rev. B 78, 085432 (2008).

72 T. Stauber and N. M. R. Peres, J. Phys. Condens. Matter 20, 055002 (2008).

73 T. Stauber, N. M. R. Peres, and A. H. Castro Neto, Phys. Rev. B 78, 085418 (2008).

74 N. M. R. Peres, T. Stauber, and A. H. Castro Neto, EPL 84, 38002 (2008).

75 L. M. Zhang, Z. Q. Li, D. N. Basov, M. M. Fogler, Z. Hao, and M. C. Martin, Phys. Rev. B 78, 235408 (2008).

${ }^{76}$ F. Hipolito, T. G. Pedersen, and V. M. Pereira, Phys. Rev. B 94, 045434 (2016).

77 J. L. Cheng, N. Vermeulen, and J. E. Sipe, Phys. Rev. B 91, 235320 (2015)

78 S. A. Mikhailov, Phys. Rev. B 93, 085403 (2016).

79 S. A. Mikhailov, Phys. Rev. B 95, 085432 (2017).

${ }^{80}$ G. B. Ventura, D. J. Passos, J. M. B. Lopes dos Santos, J. M. Lopes Viana Parente, and N. M. R. Peres, Phys. Rev. B 96, 035431 (2017).

81 D. Dimitrovski, L. B. Madsen, and T. G. Pedersen, Phys. Rev. B 95, 035405 (2017).

${ }^{82}$ R. McGouran and M. M. Dignam, Phys. Rev. B 96, 045439 (2017).

83 M. Mucha-Kruczyński, O. Tsyplyatyev, A. Grishin, E. McCann, V. I. Fal'ko, A. Bostwick, and E. Rotenberg, Phys. Rev. B 77, 195403 (2008).

84 A. Grüneis, C. Attaccalite, L. Wirtz, H. Shiozawa, R. Saito, T. Pichler, and A. Rubio, Phys. Rev. B 78, 205425 (2008).

85 A. B. Kuzmenko, I. Crassee, D. van der Marel, P. Blake, and K. S. Novoselov, Phys. Rev. B 80, 165406 (2009).

86 B. Partoens and F. M. Peeters, Phys. Rev. B 74, 075404 (2006).

87 E. McCann and V. I. Fal'ko, Phys. Rev. Lett. 96, 086805 (2006).

${ }^{88}$ L. M. Malard, J. Nilsson, D. C. Elias, J. C. Brant, F. Plentz, E. S. Alves, A. H. Castro Neto, and M. A. Pimenta, Phys. Rev. B 76, 201401 (2007).

89 A. B. Kuzmenko, E. van Heumen, D. van der Marel, P. Lerch, P. Blake, K. S. Novoselov, and A. K. Geim, Phys. Rev. B 79, 115441 (2009).

90 Z. Q. Li, E. A. Henriksen, Z. Jiang, Z. Hao, M. C. Martin, P. Kim, H. L. Stormer, and D. N. Basov, Phys. Rev. Lett. 102, 037403 (2009).

91 A. H. Castro Neto, F. Guinea, N. M. R. Peres, K. S. Novoselov, and A. K. Geim, Rev. Mod. Phys. 81, 109 (2009).

92 E. McCann and M. Koshino, Rep. Prog. Phys. 76, 056503 (2013).

93 E. V. Castro, K. S. Novoselov, S. V. Morozov, N. M. R. Peres, J. M. B. L. dos Santos, J. Nilsson, F. Guinea, A. K. Geim, and A. H. Castro Neto, Phys. Rev. Lett. 99, $216802(2006)$.

94 D. Xiao, M.-C. Chang, and Q. Niu, Rev. Mod. Phys. 82, 1959 (2010).

95 S. Haussühl, Physical Properties of Crystals (Wiley-VCH Verlag GmbH, Weinheim, Germany, 2007).

96 X.-L. Yang and S.-W. Xie, Appl. Opt. 34, 6130 (1995).

97 N. W. Ashcroft and N. D. Mermin, Solid state physics (Thomson Learning, Inc., London, 1976).

98 G. D. Mahan, Many-Particle Physics, 3rd ed. (Springer US, Boston, MA, 2000). 
99 M. P. Marder, Condensed Matter Physics, 2nd ed. (John Wiley \& Sons, Inc., Hoboken, NJ, USA, 2010).

100 S. Das Sarma, S. Adam, E. H. Hwang, and E. Rossi, Rev. Mod. Phys. 83, 407 (2011).

101 A. Säynätjoki, L. Karvonen, J. Riikonen, W. Kim, S. Mehravar, R. A. Norwood, N. Peyghambarian, H. Lipsanen, and K. Kieu, ACS Nano 7, 8441 (2013).

102 A. Autere, C. R. Ryder, A. Säynätjoki, L. Karvonen, B. Amirsolaimani, R. A. Norwood, N. Peyghambarian, K. Kieu, H. Lipsanen, M. C. Hersam, and Z. Sun, J. Phys. Chem. Lett. 8, 1343 (2017).

103 K. Alexander, N. A. Savostianova, S. A. Mikhailov, B. Kuyken, and D. Van Thourhout, ACS Photon. (2017), 10.1021/acsphotonics.7b00559.
104 B. A. Ruzicka, S. Wang, L. K. Werake, B. Weintrub, K. P. Loh, and H. Zhao, Phys. Rev. B 82, 195414 (2010).

105 K. J. Tielrooij, J. C. W. Song, S. A. Jensen, A. Centeno, A. Pesquera, A. Zurutuza Elorza, M. Bonn, L. S. Levitov, and F. H. L. Koppens, Nat. Phys. 9, 248 (2013).

106 R. I. Woodward, R. T. Murray, C. F. Phelan, R. E. P. de Oliveira, T. H. Runcorn, E. J. R. Kelleher, S. Li, E. C. de Oliveira, G. J. M. Fechine, G. Eda, and C. J. S. de Matos, 2D Mater. 4, 011006 (2016).

107 R. W. Boyd, Z. Shi, and I. De Leon, Opt. Commun. 326, 74 (2014).

108 H. Q. Le and S. Di Cecca, Opt. Lett. 16, 901 (1991).

109 M. Pu, L. Ottaviano, E. Semenova, and K. Yvind, Optica 3, 823 (2016). 